\title{
Construction of Markov Processes from Hitting Distributions
}

\author{
C. T. SHIH *
}

\section{Introduction}

As demonstrated in the fundamental memoir of Hunt [8], in the study of a general strong Markov process with nice path behavior hitting distributions play a very important role. For standard processes on a locally compact second countable Hausdorff space with the same family of hitting distributions, it is proved by Blumenthal, Getoor and McKean [2] (see also [1]) that they can be obtained, up to equivalence, from a single process by means of random time change. This suggests that a large part of the theory of Markov processes is intrinsically associated with hitting distributions rather than with transition probabilities. Thus the problem of constructing a process with given hitting distributions is a most natural one. This paper has resulted from an effort to extend the theorems of Knight and Orey [9] and Dawson [5], which deal with this problem. (The latter treats diffusion processes only.) In [11] we announced a theorem that is more general than the above theorems. All three assume given two ingredients for the construction. One is a family of measures on the state space, assumed to be locally compact second countable Hausdorff, that behave like the hitting distributions of a Markov process for a large class sets and are smooth. Smoothness means in our case that the measures transform continuous functions vanishing at infinity into such functions, which is weaker than that assumed by Knight and Orey and by Dawson in different ways. The other is a function $g$ on the state space, continuous and vanishing at infinity, with $g(x)$ meant to be the expected lifetime of the process starting at each state $x$. Knight and Orey's condition on $g$ is rather unnatural, while Dawson's and ours involve the fine topology defined from the given hitting distributions. The present result is obtained by first relaxing the condition that $g$ is required to satisfy in our earlier result, and then constructing such a function from the given hitting distributions, which now satisfy a natural transience condition in addition to the original smoothness condition. (The form of the function $g$ is suggested in [9].) This furnishes a significant extension of our previous theorem. The processes under consideration here are transient; but further extension to the construction of recurrent processes (recurrent in the sense of one- and two-dimensional Brownian motions) with smooth hitting distributions does not seem too difficult, using the theorem of [2] mentioned above.

* This research was supported by an ONR Research Associateship at Cornell University and by NSF Grant GP-6549. 
The problem of constructing a Markov process from hitting distributions also arises in the axiomatic potential theory of Brelot and Bauer. There one is given harmonic functions and therefore harmonic measures on a space, and it is natural to ask when there exists a corresponding Markov process, i.e., a process with the given harmonic measures as hitting distributions. This question has been satisfactorily answered by Meyer [10], Boboc, Constantinescu and Cornea [3], and Hansen [6]. A more general result appears in Hansen [7] (published after a first draft of this paper was submitted). This result is quite close to ours; the main difference between the two is that Hansen allows fewer "regular sets" (our sets in the family $\mathcal{O}$ appearing in the beginning of Section 1) but assumes stronger smoothness condition on these sets. It should be pointed out that $[10,3,6$ and 7$]$ use mainly potential theoretic facts.

\section{Main Results}

We consider the one-point compactification $K$ of a locally compact second countable Hausdorff space. Let $\Delta \in K$ be the point at infinity, $\rho$ be a metric on $K$, and $\mathscr{B}$ be the $\sigma$-field of Borel subsets of $K$. Let the Banach spaces of bounded real-valued Borel measurable function and real-valued continuous functions on $K$ (with the sup norm) be denoted by $\mathscr{M}$ and $\mathscr{C}$ respectively, and their subspaces of functions vanishing at $\Delta$ denoted by $\mathscr{M}_{0}$ and $\mathscr{C}_{0}$ respectively. Let $\mathcal{O}$ be a base of the topology of $K$ that is closed under the formation of finite unions and finite intersections. Let $\mathscr{D}=\{(K-U) \cup \Delta \mid U \in \mathcal{O}\}$ (we shall almost always write $\{\Delta\}$ as $\Delta$ ). Note that $\mathscr{D}$ is closed under the formation of finite unions and finite intersections.

We shall be involved in constructing Hunt processes on $K$ with $\Delta$ as the death point. Briefly, a Hunt process $\left(\Omega, X_{t}, P_{x}\right)$ is a right continuous strong Markov process satisfying the quasi-left-continuity on $[0, \infty)$ : if stopping times $T_{n}$ increase to $T$, then $X\left(T_{n}\right)$ converge to $X(T)$ almost surely on $\{T<\infty\}$. For the detailed definition and other relevant facts about Markov processes we refer to [1]. For such a process the (first) hitting time $T_{A}$ of $A \subset K$ is defined by $T_{A}(\omega)=\inf \left\{t \geqq 0 \mid X_{t}(\omega) \in A\right\}$ (which has value $\infty$ if $X_{t}(\omega) \in A$ for no $t$ ). Note that this definition is different from the usual one and should be kept in mind in reading the results of this paper. The time $T_{A}$ of reaching $\Delta$ is the lifetime. If $A$ is Borel (or even analytic), $T_{A}$ is a stopping time, so that for each $x$ in $K P_{x}\left[X\left(T_{A}\right) \in B ; T_{A}<\infty\right]$ defines a measure on $(K, \mathscr{B})$, which we shall call the hitting distribution of $A$ when the process starts at $x$.

Let $\left\{H_{D}(x, \cdot) \mid x \in K, D \in \mathscr{D}\right\}$ be a family of measures on $(K, \mathscr{B})$. We introduce the following hypotheses:

1) $H_{D}(x, \cdot)$ is a probability measure concentrated on $D$ for every $x$ and $D$.

2) $H_{D}(x, B)$ is Borel measurable in $x$ for every $D$ and (Borel) $B$.

3) $H_{D}(x, \cdot)=\varepsilon_{x}(\cdot)$, the unit mass at $x$, if $x \in D$.

4) $H_{D}(x, B)=\int H_{D^{\prime}}(x, d y) H_{D}(y, B)$ for every $x, B, D, D^{\prime}$ with $D \subset D^{\prime}$.

5) If $f \in \mathscr{C}_{0}$, then $x \rightarrow H_{D} f(x) \equiv \int H_{D}(x, d y) f(y)$ is in $\mathscr{C}_{0}$.

6) For any $D$ and $x \notin D$

$$
\lim _{\varepsilon \downarrow 0} \sup \int H_{D}(x, d y) H_{D^{\prime}}\left(y, D^{\prime}-A\right)<1
$$


where the sup is taken over all $D^{\prime}$ with $x \in D^{\prime}$ and $\operatorname{diam}\left(D^{\prime}-\Delta\right)<\varepsilon$. (Diam $A$ denotes the diameter of $A$.)

Theorem 1. Under hypotheses 1) through 6$)$, there exists a Hunt process $\left(\Omega, X_{t}, P_{x}\right)$ on $K$, with $\Delta$ as the death point, such that its hitting distribution of any $D \in \mathscr{D}$, when starting at any $x \in K$, is $H_{D}(x, \cdot)$.

The process in the theorem is not unique (up to equivalence) since the time scale is not prescribed. The next theorem deals with the construction of processes with prescribed time scale as well as prescribed hitting distributions. Let us introduce a new hypothesis:

7) There exists a nonnegative $g$ in $\mathscr{C}_{0}$ such that for any $x \in K$, neighborhood $U$ of $x$ and $\varepsilon>0$, one can find $\delta>0$ satisfying the following:

$$
\text { if } H_{D}(x, K-U)>\varepsilon, \quad \text { then } g(x)-\int H_{D}(x, d y) g(y)>\delta \text {. }
$$

Theorem 2. Under hypotheses 1) through 5) and 7), there exists a unique Hunt process $\left(\Omega, X_{t}, P_{x}\right)$ on $K$, with $\Delta$ as the death point, such that starting at any $x \in K$, its hitting distribution of any $D \in \mathscr{D}$ is $H_{D}(x, \cdot)$ and its expected lifetime equals $g(x)$.

Some remarks are in order. (i) Because of hypothesis 1 ) the space $\mathscr{C}_{0}$ may be replaced by $\mathscr{C}$ in hypothesis 5 ) (we shall often use this equivalent condition). But we choose to write it this way for the obvious reason that one may think in terms of the corresponding spaces of functions on $K-A$. (ii) Hypothesis 6) dictates that the process in Theorem 1 be transient, but it is also necessary in a transient process. Because of hypothesis 1) the process in Theorem 1 must have finite lifetime almost surely starting at any point; thus it also requires the process to be transient. However hypothesis 1) does not imply hypothesis 6); in fact it is easy to construct examples of $\left\{H_{D}(x, \cdot)\right\}$ where hypotheses 1) through 5) are satisfied but not hypothesis 6). (iii) Define from the function $g$ in hypotheses 7 )

$$
g_{D}(x)=g(x)-\int H_{D}(x, d y) g(y)=g(x)-H_{D} g(x) .
$$

For the process in Theorem 2 , this is the expected hitting time of $D$ when the process starts at $x$. Thus because of the right continuity of the process, (1.1) of hypothesis 7) is clearly a necessary condition in the theorem. (iv) Hypothesis 6) is not stated as a condition in Theorem 2, but is implicit in the other hypotheses as we shall presently see. (Hence we are entitled to use it in the proof of Theorem 2.) First it follows immediately from (1.1) upon taking $U=K-D$ and $\varepsilon=\frac{1}{2}$ that

$$
\text { if } x \notin D, \text { then } g_{D}(x)>0 \text {. }
$$

Assuming hypothesis 1) through 5) and the existence of a nonnegative $g$ in $\mathscr{C}_{0}$ satisfying (1.2) we show hypothesis 6) holds. For if not, then there exists $D$ such that for any positive $\varepsilon$ and $\delta$ one can find $D^{\prime} \in \mathscr{D}$ with $x \in D^{\prime}$, $\operatorname{diam}\left(D^{\prime}-\Delta\right)<\varepsilon$ and $\int H_{D}(x, d y) H_{D^{\prime}}\left(y, D^{\prime}-\Delta\right)>1-\delta$. But since by (1.2) and hypothesis 3$) g_{D^{\prime}}(y) \geqq 0$,

$$
g(x)-g_{D}(x) \geqq \int H_{D}(x, d y) \int H_{D^{\prime}}(y, d z) g(z) .
$$

Since $g$ is continuous, the above implies $g(x)-g_{D}(x) \leqq g(x)$, so that we have $g_{D}(x) \leqq 0$, contradicting (1.2). (v) In view of Theorem 1 and the above discussion, it might appear to be a possibility to replace (1.1) by the apparently weaker (1.2) 
in hypothesis 7) (which would imply that (1.1) and (1.2) were equivalent under other conditions of the hypothesis). However, there exist simple examples showing this is not the case.

Theorem 1 is proved in Section 2, assuming Theorem 2. The rest of the paper constitutes the proof of Theorem 2, with a sketch given in Section 3.

\section{Construction of the Function $g$}

In this section we assume hypotheses 1) through 6) and prove hypothesis 7). Thus Theorem 1 will follow from Theorem 2 . We note first an immediate consequence of hypothesis 4): for $D \subset D^{\prime}, f \in \mathscr{M}$

$$
H_{D} f(x)=\int H_{D^{\prime}}(x, d y) H_{D} f(y)=H_{D^{\prime}} H_{D} f(x) .
$$

Lemma 2.1. For any $x, D, F, H_{F}(x, F-\Delta) \geqq \int H_{D}(x, d y) H_{F}(y, F-\Delta)$.

Proof.

$$
\begin{aligned}
H_{F}(x, F-\Delta) & =\int H_{D \cup F}(x, d y) H_{F}(y, F-\Delta) \\
& =\int_{D} H_{D \cup F}(x, d y) H_{F}(y, F-\Delta)+H_{D \cup F}(x, F-D) .
\end{aligned}
$$

Since $\int H_{D}(y, d z) H_{F}(z, F-\Delta)$ equals $H_{F}(y, F-\Delta)$ for $y \in D$ and never exceeds 1 , the above

by (2.1).

$$
\begin{aligned}
& \geqq \int H_{D \cup F}(x, d y) \int H_{D}(y, d z) H_{F}(z, F-\Delta) \\
& =\int H_{D}(x, d z) H_{F}(z, F-\Delta)
\end{aligned}
$$

Note that if $D \subset F$, then from the lemma $H_{F}(x, F-\Delta) \geqq H_{D}(x, D-\Delta)$.

Lemma 2.2. Let $U \in \mathcal{O}$ be a neighborhood of $\Delta$ and $\varepsilon>0$. There is a neighborhood $V$ of $\Delta$ such that $H_{(K-U) \cup \Delta}(x, K-U)<\varepsilon$ for all $x \in V$.

Proof. Let $f \in \mathscr{C}_{0}$ be equal to 1 on $K-U$. Then $V=\left\{H_{(K-U) \cup \Delta} f<\varepsilon\right\}$ is such a neighborhood. (Remark: by a neighborhood we always mean an open one.)

We shall now define a function $g$ satisfying the conditions in hypothesis 7). Let $a_{k}$ be positive with $\sum_{k} a_{k}<\infty$, and choose $D_{k} \in \mathscr{D}$ such that each $D_{k}-\Delta$ is closed and that for any $x$ and $\varepsilon>0$, there is some $k$ with $x \in D_{k}$ and $\operatorname{diam}\left(D_{k}-\Delta\right)<\varepsilon$. Define

$$
g(x)=\sum_{k} a_{k} H_{D_{k}}\left(x, D_{k}-\Delta\right)
$$

Lemma 2.3. $g \geqq 0, g \in \mathscr{C}_{0}$, and $g_{D}(x)>0$ if $x \notin D$, where (again)

$$
g_{D}(x)=g(x)-H_{D} g(x) \text {. }
$$

Proof. Plainly $g \geqq 0$. Since $D_{k}-\Delta$ is closed there exists $f \in \mathscr{C}_{0}$ with $f=1$ on $D_{k}-\Delta$. Thus $x \rightarrow H_{D_{k}}\left(x, D_{k}-\Delta\right)=H_{D_{k}} f(x)$ is in $\mathscr{C}_{0}$ by hypothesis 5$)$. It follows that $g \in \mathscr{C}_{0}$. If $D \in \mathscr{D}$ and $x \notin D$, then by hypothesis 6) there exists $m$ such that $x \in D_{m}$ and $\int H_{D}(x, d y) H_{D_{m}}\left(y, D_{m}-\Delta\right)<1$. Now

$$
g_{D}(x)=\sum_{k} a_{k}\left[H_{D_{k}}\left(x, D_{k}-\Delta\right)-\int H_{D}(x, d y) H_{D_{k}}\left(y, D_{k}-\Delta\right)\right] .
$$


The $m$-th term in the above sum is positive since $H_{D_{m}}\left(x, D_{m}-\Delta\right)=1$, and by Lemma 2.1 each term is nonnegative. Hence $g_{D}(x)>0$.

We observe from the lemma and hypothesis 3) that $g_{F} \geqq 0$ and so $g \geqq H_{F} g$ for any $F$. It follows that if $F_{1} \subset F_{2}$

$$
g_{F_{1}}=g-H_{F_{1}} g=g-H_{F_{2}} H_{F_{1}} g \leqq g-H_{F_{2}} g=g_{F_{2}} .
$$

Let $x$ be fixed, $U$ be a neighborhood of $x$ and $\varepsilon>0$. We shall find $\delta>0$ for which (1.1) holds. Clearly we may assume $x \neq \Delta, \varepsilon<1, K-U \in \mathscr{D}$ and $\operatorname{diam} U<\rho(x, \Delta) / 2$. Let $U^{\prime}$ be a neighborhood of $\Delta$ with $U \subset K-U^{\prime}$, and let $V$ be a neighborhood of $\Delta$ such that

for all $y \in V$ (Lemma 2.2).

$$
H_{\left(K-U^{\prime}\right) \cup \Delta}\left(y, K-U^{\prime}\right)<\varepsilon
$$

Lemma 2.4. There exist $D_{m}$ containing $x$ and $\delta_{0}>0$ such that if $H_{D}(x, K-U)>\varepsilon$, then $\int H_{D}(x, d y) H_{D_{m}}\left(y, D_{m}-\Delta\right)<1-\delta_{0}$.

Proof. If $F \in \mathscr{D}$ and $F-\Delta \subset U$, then since $g_{F}=g-H_{F} g \in \mathscr{C}_{0}$ and is strictly positive on $K-F$, the infimum $\delta_{1}$ of $g_{F}$ on $K-(U \cup V)$ is positive. Since by (2.2) $g_{F}$ increase as $F$ decreases, we may choose $F=D_{m}$ such that $\delta_{1}>0, x \in D_{m}$, and $|g(x)-g(y)|<\varepsilon \delta_{1} / 4$ for $y \in D_{m}-\Delta$. We claim that for this $D_{m}$ there exists $\delta_{0}>0$ satisfying the desired condition. Suppose the contrary. Then for an arbitrary $\delta_{2}>0$ there exists $D$ such that $H_{D}(x, K-U)>\varepsilon$ and $\int H_{D}(x, d y) H_{D_{m}}\left(y, D_{m}-\Delta\right)>1-\delta_{2}$. Let $\delta_{2}<\varepsilon(1-\varepsilon) / 2$. Then, noting that

$$
H_{D_{m}}\left(y, D_{m}-4\right) \leqq H_{\left(K-U^{\prime}\right) \cup \Delta}\left(y, K-U^{\prime}\right),
$$

which follows from the remark following Lemma 2.1 since $D_{m} \subset\left(K-U^{\prime}\right) \cup \Delta$, we have

$$
\begin{aligned}
1-\varepsilon(1-\varepsilon) / 2 & <\int H_{D}(x, d y) H_{D_{m}}\left(y, D_{m}-\Delta\right) \\
& \leqq 1-H_{D}(x, V)+\int_{V} H_{D}(x, d y) H_{\left(K-U^{\prime}\right) \cup \Delta}\left(y, K-U^{\prime}\right) \\
& \leqq 1-H_{D}(x, V)+\varepsilon H_{D}(x, V)
\end{aligned}
$$

by (2.3). Hence $H_{D}(x, V)<\varepsilon / 2$ and it follows that $H_{D}(x, K-(U \cup V))>\varepsilon / 2$. Now since $g_{F} \geqq 0$ for any $F$,

$$
\begin{aligned}
g(x) & \geqq \int H_{D}(x, d y) g(y)=\int H_{D}(x, d y)\left[g_{D_{m}}(y)+\int H_{D_{m}}(y, d z) g(z)\right] \\
& \geqq\left[\delta_{1} H_{D}(x, K-(U \cup V))\right]+\left[\inf _{z \in D_{m}-\Delta} g(z)\right] \int H_{D}(x, d y) H_{D_{m}}\left(y, D_{m}-\Delta\right) \\
& >\delta_{1} \varepsilon / 2+\left(g(x)-\varepsilon \delta_{1} / 4\right)\left(1-\delta_{2}\right)>g(x)\left(1-\delta_{2}\right)+\varepsilon \delta_{1} / 4 .
\end{aligned}
$$

The last expression being greater than $g(x)$ if $\delta_{2}$ is sufficiently small, we have arrived at a contradiction.

Proposition 2.5. Hypotheses 1) through 6) imply hypothesis 7).

Proof. With the given $x, U$ and $\varepsilon$, we shall find $\delta>0$ satisfying (1.1). Let $D_{m}$ and $\delta_{0}$ be as in Lemma 2.4. Let $\delta=a_{m} \delta_{0}$. Then $\delta>0$, and if $H_{D}(x, K-U)>\varepsilon$, then

$$
\begin{aligned}
g(x)-\int H_{D}(x, d y) g(y) & \geqq a_{m}\left[H_{D_{m}}\left(x, D_{m}-\Delta\right)-\int H_{D}(x, d y) H_{D_{m}}\left(y, D_{m}-\Delta\right)\right] \\
& >a_{m}\left[1-\left(1-\delta_{0}\right)\right]=\delta .
\end{aligned}
$$




\section{A Partitioning of the State Space and a Sketch of the Proof of Theorem 2}

Definition. An ordered pair $(\mathscr{U}, \mathscr{V})$ is a partition (of $K$ ) if $\mathscr{U}=\left\{U_{1}, \ldots, U_{m}\right\}$ is an open covering of $K$ and $\mathscr{V}=\left\{V_{1}, \ldots, V_{m}\right\}$ is an (ordinary) partition of $K$ consisting of Borel sets such that $V_{i} \subset U_{i}$ for every $i$. A partition $(\mathscr{U}, \mathscr{V})$ is a refinement of another partition $\left(\mathscr{U}^{\prime}, \mathscr{V}^{\prime}\right)$, and we write $(\mathscr{U}, \mathscr{V}) \subset\left(\mathscr{U}^{\prime}, \mathscr{V}^{\prime}\right)$, if whenever $V_{i} \in V$, $U_{j}^{\prime} \in \mathscr{U}^{\prime}$ and $V_{i} \cap U_{j}^{\prime} \neq \phi$, we have $U_{i} \subset U_{j}^{\prime}$.

According to [12; Proposition 2.1] we can choose a sequence of partitions $\left(\mathscr{U}_{n}, \mathscr{V}_{n}\right)$ with $\mathscr{U}_{n}=\left\{U_{n 1}, \ldots, U_{n m_{n}}\right\}, \mathscr{V}_{n}=\left\{V_{n 1}, \ldots, V_{n m_{n}}\right\}$ such that
i) $\mathscr{U}_{n} \subset \mathcal{O}$,
ii) $\left(\mathscr{U}_{n}, \mathscr{V}_{n}\right) \subset\left(\mathscr{U}_{k}, \mathscr{V}_{k}\right)$ if $n \geqq k$,
iii) $\max _{1 \leqq i \leqq m_{n}} \operatorname{diam} U_{n i}<1 / n$,
iv) $V_{n i}=U_{n i}-\bigcup_{j<i} U_{n j}$ (in particular $V_{n 1}=U_{n 1}$ ).

The fact $\left(\mathscr{U}_{n}, \mathscr{V}_{n}\right) \subset\left(\mathscr{U}_{n}, \mathscr{V}_{n}\right)$ (not necessarily true for an arbitrary partition) implies that if $x \in V_{n i}$, then $U_{n i}$ is the intersection of all $U_{n j}$ containing $x$. We set

$$
D(n, x)=\left(K-U_{n i}\right) \cup \Delta \quad \text { if } x \in V_{n i} \text {. }
$$

Let $\mathscr{D}_{n}$ be the class of all (finite) intersections of sets of the form $(K-U) \cup \Delta$ with $U \in \mathscr{U}_{n}$, or, what is the same, the class of sets of the form $(K-U) \cup \Delta$ where $U$ is a (finite) union of sets in $\mathscr{U}_{n}$. Let $\mathscr{D}_{\infty}=\bigcup_{n} \mathscr{D}_{n}$. From i) above we have $\mathscr{D}_{\infty} \subset \mathscr{D}$; because of iii) for any $D \in \mathscr{D}$ there exist $D_{n} \in \mathscr{D}_{n}$ such that $D_{n} \downarrow D$; ii) implies the important properties that $\mathscr{D}_{n}$ increases with $n$ and that if $k \leqq n, D \in \mathscr{D}_{k}$ and $x \notin D$, then $D \subset D(n, x)$.

From now on the conditions of Theorem 2, i.e., hypotheses 1) through 5) and 7), are in force. Because of a discussion in Section 1, hypothesis 6) is also valid. Set for $x \in K, B \in \mathscr{B}$

$$
Q^{(n)}(x, B)=H_{D(n, x)}(x, B), \quad h_{n}(x)=g_{D(n, x)}(x), \quad e_{n}(x)=1 / h_{n}(x) .
$$

We now sketch the proof of Theorem 2. First we construct a sequence of (strong Markov) jump processes $X^{(n)}=\left(X_{t}^{(n)}, P_{x}^{(n)}\right)$ on $K$ satisfying the following conditions: (i) starting at a point $x \neq \Delta$ the process $X^{(n)}$ stays there for a random time $t$ according to the distribution $1-\exp \left(-e_{n}(x) t\right)\left(\right.$ i. e., $h_{n}(x)$ is the expected holding time at $x$ ); (ii) then it jumps to a new point $y$ according to the distribution $H_{D(n, x)}(x, d y)$; (iii) with $T_{\beta}$ denoting the $\beta$-th jump ( $\beta$ a countable ordinal), we have $X^{(n)}\left(T_{\alpha_{k}}\right)$ converging to $X^{(n)}\left(T_{\alpha}\right)$ almost surely whenever $\alpha_{k} \uparrow \alpha$; (iv) $\Delta$ is an absorbing point for $X^{(n)}$. From these conditions it is easily shown that the hitting distribution of any $D \in \mathscr{D}_{n}$ for $X^{(n)}$ starting at $x$ is $H_{D}(x, \cdot)$ and its expected lifetime is $g(x)$, for any $x \in K$. While referring the detailed construction of the $X^{(n)}$ to a method in $[1$; Chapter $I, \S 12]$, we do the simpler construction of their discrete skeletons $Z^{(n)}=\left(Z_{\alpha}^{(n)}, Q_{x}^{(n)}\right)$ (it suffices to let $\alpha$ range over countable ordinals less than $\omega^{\omega}$, where $\omega$ is the first infinite ordinal and $\omega^{\omega}$ is the limit of $\omega^{n}$ ), and thereby establish the crucial condition (iii) satisfied by the $X^{(n)}$. Following [5] we shall call the $Z^{(n)}$ generalized random walks; they are entirely similar to the ones constructed in that paper. 
The above is done in Section 4. In Section 5 we study the convergence of $X^{(n)}$. Let $\left\{R_{\lambda}^{(n)}, \lambda \geqq 0\right\}$ be the resolvent of $X^{(n)}$ on $\mathscr{M}$ :

$$
R_{\lambda}^{(n)} f(x)=E_{x}^{(n)} \int_{0}^{\infty} e^{-\lambda t} f\left(X_{t}^{(n)}\right) d t
$$

where $E_{x}^{(n)}$ denotes expectation with respect to $P_{x}^{(n)}$. Then we show that for $f \in \mathscr{C}_{0}$, $\alpha \geqq 0, R_{\lambda}^{(n)} f$ converges uniformly, and the limit, to be denoted by $R_{\lambda}^{(\infty)} f$, is in $\mathscr{C}_{0}$. The operators $R_{\lambda}^{(\infty)}, \lambda \geqq 0$, on $\mathscr{C}_{0}$ satisfy the following: $\left\|\lambda R_{\lambda}^{(\infty)}\right\| \leqq 1, R_{\lambda}^{(\infty)}-R_{\mu}^{(\infty)}=$ $(\mu-\lambda) R_{\lambda}^{(\infty)} R_{\mu}^{(\infty)}$, and $\lambda R_{\lambda}^{(\infty)} f \rightarrow f$ uniformly for every $f \in \mathscr{C}_{0}$. Here the difficult fact (indeed the most difficult in the whole paper) to establish is the pointwise convergence of $\lambda R_{\lambda}^{(\infty)} f$ to $f\left(f \in \mathscr{C}_{0}\right)$, to which is given a whole section-Section 6 , and for which we have to study the projective limit process of the $Z^{(n)}$. Now by the Hille-Yoshida theorem and the theorem of Blumenthal there exists a Hunt process $X^{(\infty)}=\left(X_{t}^{(\infty)}, P_{x}^{(\infty)}\right)$ on $K$ with $\left\{R_{\lambda}^{(\infty)}, \lambda \geqq 0\right\}$ as its resolvent on $\mathscr{C}_{0}$. We show that the finite dimensional distributions of $X^{(n)}$ converge weakly to the corresponding ones of $X^{(\infty)}$ under any common initial distribution, and that the processes $X^{(n)}, 1 \leqq n \leqq \infty$, satisfy a uniform regularity condition. These two facts allow us to apply a theorem of Skorokhod and prove in Section 7 that the hitting distributions of $X^{(n)}$ converge to the corresponding ones of $X^{(\infty)}$ for all $D \in \mathscr{D}_{\infty}$, which then implies that $X^{(\infty)}$ has the right hitting distributions for all $D \in \mathscr{D}$. The other properties of $X^{(\infty)}$ are easily established.

\section{Construction of $Z^{(n)}$ and $X^{(n)}$}

The main work in this section is the construction of a typical generalized random walk $Z^{(n)}$, which is the discrete skeleton of the approximating process $X^{(n)}$. Let $(\mathscr{U}, \mathscr{V})$ be a partition with $\mathscr{U}=\left\{U_{1}, \ldots, U_{m}\right\}, \mathscr{V}=\left\{V_{1}, \ldots, V_{m}\right\}$ such that $\mathscr{U} \subset \mathcal{O},(\mathscr{U}, \mathscr{V}) \subset(\mathscr{U}, \mathscr{V})$ and $V_{i}=U_{i}-\bigcup_{j<i} U_{j}$. Let $\mathscr{D}_{0}$ be the class of all (finite) intersections of sets of the form $(K-U) \cup \Delta$ with $U \in \mathscr{U}$. Set $D(x)=\left(K-U_{i}\right) \cup \Delta$ if $x \in V_{i}$. We emphasize again that if $x \in V_{i}, U_{i}$ is the intersection of all $U_{j}$ containing $x$, and so if $x \notin D$ and $D \in \mathscr{D}_{0}$, then $D \subset D(x)$. Set

$$
Q(x, B)=H_{D(x)}(x, B), \quad h(x)=g_{D(x)}(x), \quad e(x)=1 / h(x) .
$$

$Q(x, B)$ is a Markov kernel (transition probability) on $(K, \mathscr{B}) . h$ is strictly positive on $K-\Delta$ and $h(\Delta)=0$; on each $V_{i}, h=g_{\left(K-U_{i}\right) \cup \Delta}$ and is thus continuous (in general $h$ is not continuous on $K$ ).

Let $\pi$ denote the ordinal $\omega^{\omega}$. Let $\mathscr{W}$ be the product space $\prod_{\alpha<\pi} K_{\alpha}$ where each $K_{\alpha}$ is a copy of $K ; Z_{\alpha}=Z(\alpha), \alpha<\pi$, be the projections on $\mathscr{W}: Z_{\alpha}(w)=w(\alpha)$ for $w \in \mathscr{W}$; $\mathscr{H}_{\alpha}, \alpha<\pi$, be the $\sigma$-field on $\mathscr{W}$ generated by the $Z_{\beta}, \beta \leqq \alpha$ (with respect to $\mathscr{B}$ ); $\mathscr{H}_{\alpha-}, \alpha \leqq \pi$, be the $\sigma$-field on $\mathscr{W}$ generated by the $Z_{\beta}, \beta<\alpha$, and $\mathscr{H}=\mathscr{H}_{\pi-} ; \theta_{\alpha}, \alpha<\pi$, be the shift operator on $\mathscr{W}$ defined by $Z_{\beta}\left(\mathcal{O}_{\alpha}\right)=Z_{\alpha+\beta}$ for all $\beta<\pi .(\alpha+\beta$ is the $\beta$-th ordinal after $\alpha$. Note that $\pi$ has the property that for $\alpha<\pi, \alpha+\pi=\pi$.) We shall define probability measures $Q_{x}, x \in K$, on $\mathscr{H}$ so that $\left(Z_{\alpha}, Q_{x}\right)$ is a (discrete parameter) Markov process on $K$, i.e., for any $x, \alpha<\pi$, and bounded real $\mathscr{H}$-measurable $\phi$

$$
\widehat{E}_{x}\left\{\phi\left(\theta_{\alpha}\right) \mid \mathscr{H}_{\alpha}\right\}=\hat{E}_{Z(\alpha)}\{\phi\}
$$


(where $\hat{E}_{x}$ denotes expectation with respect to $Q_{x}$ ), has $Q(x, B)$ as its onestep transition probability, and satisfies the following left continuity: for $\alpha<\pi$

$$
\text { if } \alpha_{k} \uparrow \alpha \text { then } Z\left(\alpha_{k}\right) \rightarrow Z(\alpha) \text { a.s. } Q_{x} \text { for every } x \text {. }
$$

The $Q_{x}$ are defined by successive extension in the following manner. (1) On $\mathscr{H}_{0}$ let $Q_{x}\left[Z_{0} \in B\right]=\varepsilon_{x}(B)$. (2) When $Q_{x}$ has been defined on $\mathscr{H}_{\beta}$ extend it to $\mathscr{H}_{\beta+1}$ by requiring

$$
Q_{x}\left[Z_{\beta+1} \in B ; A\right] \equiv Q_{x}\left(\Lambda \cap\left\{Z_{\beta+1} \in B\right\}\right)=\int Q_{x}\left[Z_{\beta} \in d y\right] Q(y, B)
$$

for $\Lambda \in \mathscr{H}_{\beta}$. (3) Suppose that the $Q_{x}$ are defined on $\bigcup_{\alpha<\beta} \mathscr{H}_{\alpha}$ for a limit ordinal $\beta<\pi$, so that by the Kolmogorov extension theorem they are extended to $\mathscr{H}_{\beta-}$. By induction (4.1) holds if $\alpha<\beta$ and $\phi$ is $\mathscr{H}_{(\beta-\alpha)_{-}}$-measurable, where $\beta-\alpha$ is the ordinal satisfying $\alpha+(\beta-\alpha)=\beta$, and (4.2) holds if $\alpha<\beta$. Now suppose we can show the following:

$$
\text { if } \alpha_{k} \uparrow \beta \text { then } Z\left(\alpha_{k}\right) \text { converges a.s. } Q_{x} \text { for every } x \text {. }
$$

Then if $\alpha_{1}<\cdots<\alpha_{k} \uparrow \alpha \leqq \beta$, the sequence of measures $Q_{x}\left[Z\left(\alpha_{k}\right) \in \cdot\right]$ converges weakly ${ }^{1}$. Denote by $v_{x, \alpha}$ the weak limit, which is of course independent of the sequence $\left\{\alpha_{k}\right\}$. Note that for $\alpha<\beta, v_{x, \alpha}(\cdot)=Q_{x}\left[Z_{\alpha} \in \cdot\right]$. Now extend $Q_{x}$ to every $\sigma$-field generated by $Z_{\beta}$ and $\mathscr{H}_{\alpha}$, where $\alpha<\beta$, by requiring

$$
Q_{x}\left[Z_{\beta} \in B ; A\right]=\int Q_{x}\left[Z_{\alpha} \in d y ; \Lambda\right] v_{y, \beta-\alpha}(B)
$$

for $\Lambda \in \mathscr{H}_{\alpha}$. Because of (4.1) (for $\mathscr{H}_{(\beta-\alpha)-}$-measurable $\phi$ ) these extensions are compatible. Hence each $Q_{x}$ has an extension to $\mathscr{H}_{\beta}$; moreover (4.1) is satisfied if $\alpha<\beta$ and $\phi$ is $\mathscr{H}_{\beta-\alpha}$-measurable. (4) If $Q_{x}$ is defined on $\bigcup_{\beta<\pi} \mathscr{H}_{\beta}$, then again by the Kolmogorov extension theorem it can be extended to all of $\mathscr{H}$. Hence in order to obtain the $Q_{x}$ we need only prove (4.3) under the assumption that they have been defined on $\mathscr{H}_{\beta-}$ as probability measures and satisfy (4.1) for $\alpha<\beta$ and $\mathscr{H}_{(\beta-\alpha)-}$-measurable $\phi$ and (4.2) for $\alpha<\beta$ (from here through Proposition 4.3 these restrictions will be implicit when we refer to (4.1) and (4.2)). Clearly we may also assume $\beta$ is such that $\beta-\alpha=\beta$ (i.e., $\alpha+\beta=\beta$ ) for all $\alpha<\beta$; for otherwise (4.3) is a trivial consequence of (4.1) and (4.2).

For $A \subset K$ let $\tau_{A}(w)=\inf \left\{\alpha<\beta \mid Z_{\alpha}(w) \in A\right\}$ if there is such $\alpha$, and $=\beta$ otherwise. This definition will be changed after Proposition 4.3 is proved. $\tau_{A}$ is the (first) hitting time of $A$ for the generalized random walk $\left(Z_{\alpha}\right)_{\alpha<\beta}$. Let

$$
H_{A}^{*}(x, B)=Q_{x}\left[Z\left(\tau_{A}\right) \in B ; \tau_{A}<\beta\right] \quad \text { for } A \in \mathscr{B}, B \in \mathscr{B} .
$$

Lemma 4.1. For any $x$ and $F \in \mathscr{D}$ with $F-\Delta$ closed, $H_{F}^{*}(x, F-\Delta) \leqq H_{F}(x, F-\Delta)$.

Proof. We show by induction

$$
H_{F}(x, F-\Delta) \geqq Q_{x}\left[Z\left(\tau_{F}\right) \in F-\Delta ; \tau_{F}<\alpha\right]+\hat{E}_{x}\left\{H_{F}\left(Z_{\alpha}, F-\Delta\right) ; \tau_{F} \geqq \alpha\right\}
$$

${ }^{1}$ Finite measures $v_{n}$ on $K$ converge weakly to a finite measure $v$ if $\int f d v_{n} \rightarrow \int f d v$ for every $f \in \mathscr{C}$. If $v_{n}, v$ are probability measures, this is equivalent to $\int f d v_{n} \rightarrow \int f d v$ for every $f \in \mathscr{C}_{0}$. 
for all $\alpha<\beta$. For $\alpha=0$ this is trivial. The one-step induction is because

$$
\begin{aligned}
& \hat{E}_{x}\left\{H_{F}\left(Z_{\alpha}, F-\Delta\right) ; \tau_{F} \geqq \alpha\right\} \geqq Q_{x}\left[Z\left(\tau_{F}\right) \in F-\Delta ; \tau_{F}=\alpha\right] \\
&+\hat{E}_{x}\left\{\int H_{D\left(Z_{\alpha}\right)}\left(Z_{\alpha}, d y\right) H_{F}(y, F-\Delta) ; \tau_{F}>\alpha\right\} \\
&= Q_{x}\left[Z\left(\tau_{F}\right) \in F-\Delta ; \tau_{F}=\alpha\right]+\hat{E}_{x}\left\{H_{F}\left(Z_{\alpha+1}, F-\Delta\right) ; \tau_{F} \geqq \alpha+1\right\}
\end{aligned}
$$

in view of Lemma 2.1. If (4.4) holds for a sequence of ordinals $\alpha$ increasing to $\gamma<\beta$ then since $H_{F}(y, F-A)$ is continuous in $y,(4.2)$ implies that (4.4) holds with $\gamma$ replacing $\alpha$. Now the desired inequality follows by letting $\alpha$ increasing to $\beta$ in (4.4).

Lemma 4.2. Suppose that (4.3) does not hold. Then there exist distinct points $x_{1}$ and $x_{2}, x_{1}$ not equal to $A$, such that for any $F_{1}, F_{2} \in \mathscr{B}$ with $x_{i}$ in the interior of $F_{i}$, $i=1,2, \sup _{x \in F_{1}-\Delta} \int H_{F_{2}}^{*}(x, d y) H_{F_{1}}^{*}\left(y, F_{1}-\Delta\right)=1$.

Proof. If (4.3) fails it is easily seen that for some $z$ there exist distinct $x_{1}$ and $x_{2}$, $x_{1} \neq \Delta$, such that for any $\delta>0$ one can find $A$ with $Q_{z}(\Lambda)>0$ and $\rho\left(x_{i}, Z_{\alpha}(w)\right)<\delta$ for infinitely many $\alpha<\beta$ for each $i=1,2$ if $w \in \Lambda$. Hence for $F_{1}, F_{2} \in \mathscr{B}$ with $x_{i}$ in the interior of $F_{i}, i=1,2$, there exists $A$ with $Q_{z}(\Lambda)>0$ such that $Z_{\alpha}(w) \in F_{i}$ for infinitely many $\alpha<\beta$ for each $i=1,2$ if $w \in \Lambda$. From the Markov property (4.1) (or indeed from the strong Markov property derived from it) it is now a routine matter to obtain the desired equality (noting that $\Delta$ is an absorbing point because of $Q(\Delta, \Delta)=1$ and $(4.2)$ ).

Let $x_{1}$ be arbitrary. Let $D, F_{1} \in \mathscr{D}$ with $x_{1} \notin D, x_{1} \in F_{1}-\Delta$ and $F_{1}-\Delta$ closed. Then since $H_{F_{1}}\left(y, F_{1}-\Delta\right)$ is continuous in $y$ and therefore $\int H_{D}(x, d y) H_{F_{1}}\left(y, F_{1}-\Delta\right)$ is continuous in $x$, it follows from hypothesis 6$)$ and the fact $H_{F_{1}}\left(y, F_{1}-\Delta\right)$ decreases with $F_{1}$ that there exist $\varepsilon>0, \delta>0$ for which

if $\operatorname{diam}\left(F_{1}-\Delta\right)<\delta$.

$$
\sup _{x \in F_{1}-\Delta} \int H_{D}(x, d y) H_{F_{1}}\left(y, F_{1}-\Delta\right)<1-\varepsilon
$$

Proposition 4.3. (4.3) is valid.

Proof. Suppose the contrary. Let $x_{1}, x_{2}$ be as in Lemma 4.2. Choose $D \in \mathscr{D}$ such that $x_{1} \notin D$ and $x_{2}$ is in the interior of $D$. If $F_{1}, F_{2} \in \mathscr{D}$ with $F_{2} \subset D$ then from Lemma 2.1 and (2.1)

$$
\begin{aligned}
\int H_{D}(x, d y) H_{F_{1}}\left(y, F_{1}-\Delta\right) & \geqq \int H_{D}(x, d y) \int H_{F_{2}}(y, d z) H_{F_{1}}\left(z, F_{1}-\Delta\right) \\
& =\int H_{F_{2}}(x, d z) H_{F_{1}}\left(z, F_{1}-\Delta\right) .
\end{aligned}
$$

Let $\varepsilon>0, \delta>0$ be such that if $F_{1} \in \mathscr{D}, x_{1} \in F_{1}, F_{1}-\Delta$ is closed and $\operatorname{diam}\left(F_{1}-\Delta\right)<\delta$, then $\sup _{x \in F_{1}-\Delta} \int H_{D}(x, d y) H_{F_{1}}\left(y, F_{1}-\Delta\right)<1-\varepsilon$. Choose such $F_{1}$ with $x_{1}$ in the interior of $F_{1}-\Delta$. Then choose $F_{2} \in \mathscr{D}$ such that $F_{2} \subset D, x_{2}$ is in the interior of $F_{2}$, and $\left|H_{F_{1}}\left(y_{1}, F_{1}-\Delta\right)-H_{F_{1}}\left(y_{2}, F_{1}-\Delta\right)\right|<\varepsilon / 2$ for $y_{1}, y_{2} \in F_{2}$. By Lemma 4.1

$$
\begin{aligned}
\int H_{F_{2}}(x, d y) H_{F_{1}}\left(y, F_{1}-\Delta\right) \geqq \sup _{y \in F_{2}} H_{F_{1}}\left(y, F_{1}-\Delta\right)-\varepsilon / 2 \\
\quad \geqq \sup _{y \in F_{2}} H_{F_{1}}^{*}\left(y, F_{1}-\Delta\right)-\varepsilon / 2 \geqq \int H_{F_{2}}^{*}(x, d y) H_{F_{1}}^{*}\left(y, F_{1}-\Delta\right)-\varepsilon / 2
\end{aligned}
$$


for any $x$. From (4.5) we then obtain

$$
\int H_{F_{2}}^{*}(x, d y) H_{F_{1}}^{*}\left(y, F_{1}-\Delta\right) \leqq \int H_{D}(x, d y) H_{F_{1}}\left(y, F_{1}-\Delta\right)+\varepsilon / 2<1-\varepsilon / 2
$$

for $x \in F_{1}-\Delta$. But this contradicts Lemma 4.2.

(4.3) being proved, we have completed the construction of the generalized random walk $Z=\left(Z_{\alpha}, Q_{x}\right)$. Since its time parameter is discrete (and even takes on only countably many values) $Z$ is strong Markov. We now redefine $\tau_{A}, A \subset K$, by setting $\tau_{A}(w)=\inf \left\{\alpha<\pi \mid Z_{\alpha}(w) \in A\right\}$ if there is such $\alpha$, and $=\pi$ otherwise. For the reason of simplying writing we define $Z_{\pi}$ and $\theta_{\pi}$ on $W$ as follows: $Z_{\pi} \equiv \Delta ; \theta_{\pi} w$ for any $w$ is the unique $w^{\prime} \in W$ satisfying $Z_{\alpha}\left(w^{\prime}\right)=\Delta$ for all $\alpha<\pi$.

Lemma 4.4. $\sum_{\alpha<\pi} \hat{E}_{x}\left\{h\left(Z_{\alpha}\right)\right\} \leqq g(x)$ for every $x$. (An infinite sum of nonnegative terms of course has an unmistakable meaning.)

Proof. Recalling $h(y)=g_{D(y)}(y)=g(y)-\int H_{D(y)}(y, d z) g(z)$ we have $g(y)=h(y)+$ $\hat{E}_{y}\left\{g\left(Z_{1}\right)\right\}$. Hence by an induction similar to that in the proof of Lemma 4.1 (noting $g$ is continuous)

$$
\begin{aligned}
g(x) & =h(x)+\hat{E}_{x}\left\{h\left(Z_{1}\right)+\hat{E}_{Z_{1}}\left[g\left(Z_{1}\right)\right]\right\}=\sum_{\alpha<2} \hat{E}_{x}\left\{h\left(Z_{\alpha}\right)\right\}+\hat{E}_{x}\left\{g\left(Z_{2}\right)\right\} \\
& =\cdots=\sum_{\alpha<\gamma} \hat{E}_{x}\left\{h\left(Z_{\alpha}\right)\right\}+\hat{E}_{x}\left\{g\left(Z_{\gamma}\right)\right\}
\end{aligned}
$$

for any $\gamma<\pi$. The lemma follows since $g \geqq 0$.

The next proposition explains why we need not define $Z_{\alpha}$ for $\alpha>\pi$. It is similar to a result in [5] and the proof is the same.

Proposition 4.5. $Q_{x}\left[\tau_{\Delta} \leqq \omega^{m}\right]=1$ for every $x$. (Recall that $m$ is the cardinality of $\mathscr{U}$ and $\omega$ is the first infinite ordinal.)

Proof. We claim that $Z_{\omega} \in(K-U) \cup \Delta$ a.s. $Q_{x}$. For if not, then because of the fact that $Z_{n} \rightarrow Z_{\omega}$ a.s. $Q_{x}$ (see (4.2)) there exist $\Lambda$ with $Q_{x}(\Lambda)>0$ and $\varepsilon>0$ such that on $A, h\left(Z_{n}\right)=g_{\left(K-U_{1}\right) \cup A}\left(Z_{n}\right)>\varepsilon$ for all sufficiently large $n$. Thus we have $\hat{E}_{x}\left\{\sum_{n<\omega} h\left(Z_{n}\right)\right\}=\infty$, contradicting Lemma 4.4. Similarly one shows that $Z(2 \omega)$, $Z(3 \omega), \ldots$ are in $\left(K-U_{1}\right) \cup \Delta$ a.s. $Q_{x}$. From the same reasoning it follows that $Z\left(\omega^{2}\right) \in\left(K-U_{1}-U_{2}\right) \cup \Delta$ a.s. $Q_{x}$ and in general $Z\left(\omega^{i}\right) \in\left(K-\bigcup_{j \leqq i} U_{j}\right) \cup \Delta$ a.s. $Q_{x}$.
Hence $Z\left(\omega^{m}\right)=\Delta$ a.s. $Q_{x}$.

Since $\Delta$ is an absorbing point, the above proposition implies that almost all paths stay at $\Delta$ from time $\omega^{m}$ on. The next proposition is now immediate from the proof of Lemma 4.4 since $g(\Delta)=0$.

Proposition 4.6. $\hat{E}_{x}\left\{\sum_{\alpha<\pi} h\left(Z_{\alpha}\right)\right\}=\sum_{\alpha<\pi} \hat{E}_{x}\left\{h\left(Z_{\alpha}\right)\right\}=g(x)$ for every $x$.

Proposition 4.7. For any $x$ and $D \in \mathscr{D}_{0}, Q_{x}\left[Z\left(\tau_{D}\right) \in \cdot\right]=H_{D}(x, \cdot)$.

Proof. By an induction similar to that in the proof of Lemma 4.1 (and Lemma 4.4) we can show

$$
H_{D} f(x)=\widehat{E}_{x}\left\{f\left(Z\left(\tau_{D}\right)\right) ; \tau_{D} \leqq \alpha\right\}+\widehat{E}_{x}\left\{H_{D} f\left(Z_{\alpha}\right) ; \tau_{D}>\alpha\right\}
$$


for a fixed $f \in \mathscr{C}$ and all $\alpha<\pi$, using the continuity of $H_{D} f$, the fact $H_{D} f=f$ on $D$, and the following fact: if $y \notin D$ then since $D \subset D(y)$

$$
H_{D} f(y)=\int H_{D(y)}(y, d z) H_{D} f(z)=\int Q(y, d z) H_{D} f(z)=\widehat{E}_{y}\left\{H_{D} f\left(Z_{1}\right)\right\} .
$$

The proposition follows from (4.6) and Proposition 4.5.

Proposition 4.8. For any $x$ and $D \in \mathscr{D}_{0}, \widehat{E}_{x}\left\{\sum_{\alpha<\tau_{D}} h\left(Z_{\alpha}\right)\right\}=g_{D}(x)$.

Proof.

$$
\begin{aligned}
g_{D}(x) & =g(x)-\int H_{D}(x, d y) g(y) \\
& =\widehat{E}_{x}\left\{\sum_{\alpha<\pi} h\left(Z_{\alpha}\right)\right\}-\hat{E}_{x}\left\{\hat{E}_{Z\left(\tau_{\boldsymbol{D}}\right)}\left[\sum_{\alpha<\pi} h\left(Z_{\alpha}\right)\right]\right\} \\
& =\hat{E}_{x}\left\{\sum_{\alpha<\pi} h\left(Z_{\alpha}\right)\right\}-\widehat{E}_{x}\left\{\sum_{\tau_{D} \leqq \alpha<\pi} h\left(Z_{\alpha}\right)\right\} \\
& =\hat{E}_{x}\left\{\sum_{\alpha<\tau_{D}} h\left(Z_{\alpha}\right)\right\}
\end{aligned}
$$

by Propositions 4.6 and 4.7 , the strong Markov property and the fact that $\tau_{D} \leqq \tau_{A}<\pi$ a.s. $Q_{x}$.

We now claim that there is a strong Markov process $X=\left(X_{t}, P_{x}\right)$ on $K$ with continuous parameter and satisfying the following: (i) starting at a point $x \neq A$ it stays there for an exponentially distributed time with mean $h(x)$; (ii) then it jumps to a new point $y$ according to the distribution $H_{D(x)}(x, d y)$; (iii) with $T_{\beta}$ denoting the $\beta$-th jump ( $\beta$ a countable ordinal), we have $X\left(T_{\alpha_{k}}\right)$ converges to $X\left(T_{\alpha}\right)$ almost surely whenever $\alpha_{k} \uparrow \alpha$; (iv) $\Delta$ is an absorbing point. For the reader who is unwilling to take the above claim on faith there are two ways to construct $X$. One is to define first a resolvent $\left\{R_{\lambda}, \lambda \geqq 0\right\}$ on $\mathscr{M}_{0}$ by

$$
R_{\lambda} f(x)=\sum_{\alpha<\pi} \hat{E}_{x}\left\{f\left(Z_{\alpha}\right)\left(\prod_{\beta<\alpha} \frac{e\left(Z_{\alpha}\right)}{\lambda+e\left(Z_{\beta}\right)}\right) \frac{1}{\lambda+e\left(Z_{\alpha}\right)}\right\}
$$

where (recalling $e=1 / h) \frac{e(\Delta)}{\lambda+e(\Delta)}$ is interpreted as 0 and the product factor in the integrand is defined to be 1 when $\alpha=0$ and the infimum of all finite "sub-products" if $\alpha \geqq \omega$. One can show directly that the resolvent equation is satisfied and $\left\|\lambda R_{\lambda}\right\| \leqq 1$. Also $R_{\lambda}\left(\mathscr{C}_{0}\right) \subset \mathscr{C}_{0}$ and $\lambda R_{\lambda} f$ converges uniformly to $f$ as $\lambda \rightarrow \infty$ for all $f \in \mathscr{C}_{0}$. Consequently there exists a Hunt process on $K$ with $\Delta$ as the death point and with $\left\{R_{\lambda}, \lambda \geqq 0\right\}$ as its resolvent on $\mathscr{M}_{0}$. One then shows that this process is just a process $X$ described above. However, this construction is rather long and therefore we avoid it. The fact $R_{\lambda}\left(\mathscr{C}_{0}\right) \subset \mathscr{C}_{0}$ is essential in this construction but the proof of it alone is complicated; of course if we should be allowed to use this fact Proposition 5.2 would be immediate from Proposition 5.1; however the proof of Proposition 5.2 is much shorter than that of $R_{0}\left(\mathscr{C}_{0}\right) \subset \mathscr{C}_{0}$.

The other construction is an extension of that of [1; Chapter I, $\S 12]$, which constructs general jump processes stopped after a sequence of jumps. We define first a generalized random walk $\left(Y_{\alpha}\right)_{\alpha<\pi}$ on the state space $K \times[0, \infty)$ with one- 
step transition probability

$$
P(x, t ; d y, d s)= \begin{cases}0, & s<t, \\ Q(x, d y) e(x) \exp [-e(x)(s-t)] d s, & s \geqq t\end{cases}
$$

(where $e(\Delta) \exp [-e(\Delta)(s-t)] d s$ stands for the unit mass at $t$ ), and satisfying the property that if $\alpha_{k} \uparrow \alpha$ then $Y_{\alpha_{k}} \rightarrow Y_{\alpha}$ (in the product topology of $K \times[0, \infty)$ ) almost surely given any initial distribution. This latter property follows easily from the corresponding property of $\left(Z_{\alpha}\right)$ and Proposition 4.6. Let $\tilde{Z}_{\alpha}$ be the first component of $Y_{\alpha}$ and $T_{\alpha}$ be its second component. By deleting a null set we may assume that if $\alpha<\beta$ then $T_{\alpha} \leqq T_{\beta}$, with equality holding only if $\tilde{Z}_{\alpha}=\Delta$. Now define

$$
X_{t}=\left\{\begin{array}{l}
\tilde{Z}_{\alpha} \text { if } T_{\alpha} \leqq t<T_{\alpha+1}, \\
\Delta \text { if } \tilde{Z}_{\alpha}=\Delta \text { and } T_{\alpha} \leqq t \text { for some } \alpha \text { or if } T_{\alpha}<t \text { for all } \alpha<\pi .
\end{array}\right.
$$

Let $P_{x}$ be the probability measure for the generalized random walk $\left(Y_{\alpha}\right)$ starting at $(x, 0)$. Then $X=\left(X_{t}, P_{x}\right)$ is the desired jump process. The proof of this fact is a straightforward extension of the argument in [1] and there is no need to give it here (inductions on $n$ are to be replaced by those on $\alpha$ and kernels $Q_{\lambda}^{\alpha}(x, B)$, $\lambda \geqq 0$, are to be defined for all $\alpha<\pi$ by setting

$$
Q_{\lambda}^{1}(x, B)=\frac{e(x)}{\lambda+e(x)} Q(x, B), \quad Q_{\lambda}^{\alpha+1}(x, B)=\int Q_{\lambda}^{\alpha}(x, d y) Q_{\lambda}^{1}(y, B),
$$

and $Q_{\lambda}^{\alpha}(x, \cdot)$ to be the weak limit of $Q_{\lambda}^{\alpha_{k}}(x, \cdot)$ where $\left.\alpha_{k} \uparrow \alpha\right)$. Also the argument in [1] shows that $X$ is in fact a Hunt process; of course for this the left continuity of $\left(Y_{\alpha}\right)$ is needed.

Now corresponding to each partition $\left(\mathscr{U}_{n}, \mathscr{V}_{n}\right)$ chosen in Section 2 there are a generalized random walk $Z^{(n)}$ and a jump process $X^{(n)} ; X^{(n)}$ satisfies the conditions stated in Section 3 and $Z^{(n)}$ is (up to equivalence) the discrete sekeleton of $X^{(n)}$. We may, and we will, assume that the sample space for all the $X^{(n)}$ is the following function space

$\Omega=\{\omega:[0, \infty) \rightarrow K \mid \omega$ is right continuous, with left limits on $(0, \infty)$

$$
\text { and } \omega(t)=\Delta \Rightarrow \omega(s)=\Delta \text { for } s \geqq t\}
$$

and $X_{t}^{(n)}(\omega)=X_{t}(\omega)=\omega(t)$. $\left(\theta_{t}\right.$ will denote the shifts of $\left(X_{t}\right)$ and we shall let $X_{\infty} \equiv \Delta$ and $\theta_{\infty} \equiv \omega_{\Delta}$ where $\omega_{\Delta}(t) \equiv \Delta$.) Thus we write $X^{(n)}=\left(X_{t}, P_{x}^{(n)}\right)$. Also by construction the $Z^{(n)}$ have the same sample space $\mathscr{W}$ and $Z_{\alpha}^{(n)}(w)=Z_{\alpha}(w)=w(\alpha)$, and so we write $Z^{(n)}=\left(Z_{\alpha}, Q_{x}^{(n)}\right)$. The hitting time of $A \in \mathscr{B}$ for the processes $X^{(n)}$ will be denoted by $T_{A}: T_{A}(\omega)=\inf \left\{t \geqq 0 \mid X_{t}(\omega) \in A\right\}$ if there is such $t$ and $=\infty$ otherwise. Let $H_{A}^{(n)}(x, B)=E_{x}^{(n)}\left\{X\left(T_{A}\right) \in B\right\}$ where $E_{x}^{(n)}$ stands for expectation with respect to $P_{x}^{(n)}$. Then from Propositions 4.6, 4.7 and 4.8 we have the following facts for any $x$ and $D \in \mathscr{D}_{n}$ :

$$
\begin{aligned}
& H_{D}^{(n)}(x, \cdot)=Q_{x}^{(n)}\left[Z\left(\tau_{D}\right) \in \cdot\right]=H_{D}(x, \cdot), \\
& E_{x}^{(n)}\left\{T_{\Delta}\right\}=\widehat{E}_{x}^{(n)}\left\{\sum_{\alpha<\pi} h_{n}\left(Z_{\alpha}\right)\right\}=g(x), \\
& E_{x}^{(n)}\left\{T_{D}\right\}=\widehat{E}_{x}^{(n)}\left\{\sum_{\alpha<\tau_{D}} h_{n}\left(Z_{\alpha}\right)\right\}=g_{D}(x),
\end{aligned}
$$


where $\widehat{E}_{x}^{(n)}$ stands for expectation with respect to $Q_{x}^{(n)}$. Let $\left\{R_{z_{0}}^{(n)}, \lambda \geqq 0\right\}$ denote the resolvent of $X^{(n)}$ on $\mathscr{H}_{0}$ :

$$
R_{\lambda}^{(n)} f(x)=E_{x}^{(n)} \int_{0}^{\infty} e^{-\lambda t} f\left(X_{t}\right) d t .
$$

Then $R_{0}^{(n)} f(x)=\sum_{\alpha<\pi} \hat{E}_{x}^{(n)}\left\{f\left(Z_{\alpha}\right) h_{n}\left(Z_{\alpha}\right)\right\}$. It follows from (4.9) that $\left\|R_{0}^{(n)}\right\|=\|g\|=$ sup $g$. Hence $\left\|R_{\lambda}\right\| \leqq \min \{1 / \lambda,\|g\|\}$. The following fact will also be used: for $D \in \mathscr{Q}_{n}$,

$$
E_{x}^{(n)}\left\{e^{-\lambda T_{D}}\right\}=\hat{E}_{x}^{(n)}\left\{\prod_{\alpha<\tau_{D}} \frac{e_{n}\left(Z_{\alpha}\right)}{\lambda+e_{n}\left(Z_{\alpha}\right)}\right\},
$$

which is obtained from an easy induction (see also (4.7)).

\section{Convergence of the Processes $X^{(n)}$}

Proposition 5.1. For $f \in \mathscr{C}_{0},\left\{R_{0}^{(n)} f, n \geqq 1\right\}$ is a Cauchy sequence in $\mathscr{M}_{0}$.

Proof. Given $\varepsilon>0$ let $N>0$ be such that if $\rho(x, y)<1 / N$ then $|f(x)-f(y)|<\varepsilon$. Let $k>n \geqq N$. Define a stopping time $R$ on $\left(\Omega, X_{t}\right)$ by setting $R=T_{D(n, x)}$ if $X_{0}=x$. Let $R_{\alpha}, \alpha<\pi$, be the iterates of $R$, i.e., $R_{0}=0, R_{\alpha+1}=R_{\alpha}+R\left(\theta_{R_{\alpha}}\right)$, and $R_{\alpha}=\sup R_{\beta}$ for a limit ordinal $\alpha$. Then by the strong Markov property for any $x$

$$
R_{0}^{(k)} f(x)=\sum_{\alpha<\pi} E_{x}^{(k)}\left\{E_{X\left(R_{\alpha}\right)}^{(k)} \int_{0}^{R} f\left(X_{t}\right) d t\right\}
$$

and the same equality holds when $k$ is replaced by $n$. Now since $\mathscr{D}_{n} \subset \mathscr{D}_{k}$ an induction based on (4.8) shows $E_{x}^{(k)}\left\{X\left(R_{\alpha}\right) \in \cdot\right\}=E_{x}^{(n)}\left\{X\left(R_{\alpha}\right) \in \cdot\right\}$ for all $\alpha$. Next since for $t<R, X_{t}$ and $X_{0}$ are in the same $U_{n i}$ and therefore $\left|f\left(X_{t}\right)-f\left(X_{0}\right)\right|<\varepsilon$, and since $R$ is the first jumping time a.s. $P_{y}^{(n)}$, we obtain from (4.10)

$$
\left|E_{y}^{(k)} \int_{0}^{R} f\left(X_{t}\right) d t-E_{y}^{(n)} \int_{0}^{R} f\left(X_{t}\right) d t\right|=\left|E_{y}^{(k)} \int_{0}^{R} f\left(X_{t}\right) d t-f(y) g_{D(n, y)}(y)\right|<\varepsilon E_{y}^{(k)}\{R\}
$$
for all $y$. Thus we have $\left|R_{0}^{(k)} f(x)-R_{0}^{(n)} f(x)\right|<\sum_{\alpha<\pi} E_{x}^{(k)}\left\{\varepsilon E_{X\left(R_{\alpha}\right)}^{(k)}[R]\right\}=\varepsilon g(x) \leqq \varepsilon\|g\|$,
proving the proposition.

Definition. For $f \in \mathscr{C}_{0}$, let $R_{0}^{(\infty)} f=\lim _{n} R_{0}^{(n)} f$.

Proposition 5.2. For $f \in \mathscr{C}_{0}, R_{0}^{(\infty)} f \in \mathscr{C}_{0}$.

Proof. Since $\left|R_{0}^{(n)} f(x)\right| \leqq g(x)\|f\|, R_{0}^{(n)} f(\Delta)=0$ and $R_{0}^{(n)} f$ is continuous at $\Delta$. We now assume $x_{0} \neq \Delta$ and $x_{l} \rightarrow x_{0}$ and show that $\overline{\lim }_{l}\left|R_{0}^{(\infty)} f\left(x_{l}\right)-R_{0}^{(x)} f\left(x_{0}\right)\right|$ is arbitrarily small. Let $\varepsilon>0$ be fixed. Choose $\delta>0$ such that if $\rho(x, \Delta)<\delta$ then $g(x)<\varepsilon$ and if $\rho(x, y)<\delta$ then $|f(x)-f(y)|<\varepsilon$. Let $N>2 / \delta$. Then if $U \in \mathscr{U}_{N}$ we have $\operatorname{diam} U<\delta / 2$. Let $V=\left\{x \mid \rho(x, \Delta)<\delta_{1}\right\}$ where $\delta / 2<\delta_{1}<\delta$ and $P_{x_{0}}^{(n)}\left[X\left(T_{\alpha}\right) \in V^{*}\right]=0$ for all $n$ and $\alpha<\pi$; here $T_{\alpha}$ is the $\alpha$-th jumping time and $V^{*}$ denote the boundary of $V$. Next for each $x \notin V$ let $U_{x}=U_{N i}$ if $x \in V_{N i}$ (note $\Delta \notin U_{x}$ ) and let $W_{x}$ be a neighborhood of $x$ with closure $\bar{W}_{x} \subset U_{x}$ and $P_{x_{0}}^{(n)}\left[X\left(T_{\alpha}\right) \in W_{x}^{*}\right]=0$ for all $n$ and $\alpha<\pi$. Now finitely many of the $W_{x}$, say $W_{1}, \ldots, W_{m}$, cover $K-V$. Let the corresponding $U_{x}$ be denoted by $U_{1}, \ldots, U_{m}$. Set $G_{i}=W_{i}-\bigcup_{j<i}^{m} W_{j}-V, i=1, \ldots, m$. Then $V, G_{1}, \ldots, G_{m}$ 
form a partition of $K ; P_{x_{0}}^{(n)}\left[X\left(T_{\alpha}\right) \in V^{*} \cup G_{1}^{*} \cup \cdots \cup G_{m}^{*}\right]=0$ for all $n$ and $\alpha$; and the distance between $G_{i}$ and $K-U_{i}=\left(K-U_{i}\right) \cup \Delta$ is positive, so that the function

$$
\begin{aligned}
h(x) & =g_{K-U_{i}}(x), \quad x \in G_{i}, 1 \leqq i \leqq m \\
& =g(x), \quad x \in V
\end{aligned}
$$

has a positive infimum $c$ on $K-V$. Define a stopping time $S$ as follows: $S=$ $T_{K-U_{i}}$ if $X_{0} \in G_{i}, 1 \leqq i \leqq m$, and $S=T_{\Delta}$ if $X_{0} \in V$. Then $h(x)=E_{x}^{(n)}\{S\}$ for $n \geqq N$. Let $S_{k}, k \geqq 0$, be the iterates of $S$. We show that for $n \geqq N$ and $k \geqq 0, P_{x_{l}}^{(n)}\left[X\left(S_{k}\right) \in \cdot\right]$ converges weakly to $P_{x_{0}}^{(n)}\left[X\left(S_{k}\right) \in \cdot\right]$ as $l \rightarrow \infty$, by induction on $k$. The case $k=0$ is trivial. Assume the convergence holds for an arbitrary $k$. Then since $P_{x_{0}}^{(n)}\left[X\left(S_{k}\right) \in\right.$ $\left.V^{*} \cup G_{1}^{*} \cup \cdots \cup G_{m}^{*}\right]=0$ (the fact $K-U_{i} \in \mathscr{D}_{N} \subset \mathscr{D}_{n}$ implies $S_{k}(\omega)=T_{\alpha}(\omega)$ for some $\alpha)$ it is easily seen that

$$
E_{x_{l}}^{(n)}\left\{f_{1}\left(X\left(S_{k}\right)\right)\right\} \rightarrow E_{x_{0}}^{(n)}\left\{f_{1}\left(X\left(S_{k}\right)\right)\right\}
$$

for any $f_{1} \in \mathscr{M}_{0}$ continuous on each of the sets $V, G_{1}, \ldots, G_{m}$. Now for any $f_{2} \in \mathscr{C}_{0}$ the function $f_{1}$ defined by $f_{1}(x)=H_{K-U_{i}} f_{2}(x)$ for $x \in G_{i},=H_{\{4\}} f_{2}(x)=0$ for $x \in V$ is such a function. But for this $f_{1}(5.1)$ becomes $E_{x_{1}}^{(n)}\left\{f_{2}\left(X\left(S_{k+1}\right)\right)\right\} \rightarrow E_{x_{0}}^{(n)}\left\{f_{2}\left(X\left(S_{k+1}\right)\right)\right\}$, establishing the induction step. Now if $n \geqq N, f_{1} \in \mathscr{M}_{0}$

$$
R_{0}^{(n)} f_{1}(x)=\sum_{k=0}^{k^{\prime}} E_{x}^{(n)}\left\{E_{X\left(\mathrm{~S}_{k}\right)}^{(n)} \int_{0}^{S} f_{1}\left(X_{t}\right) d t\right\}+E_{x}^{(n)}\left\{R_{0}^{(n)} f_{1}\left(X\left(S_{k^{\prime}+1}\right)\right)\right\}
$$

and with $f_{1}=1_{K-\Delta}$ we have

$$
g(x)=\sum_{k=0}^{k^{\prime}} E_{x}^{(n)}\left\{h\left(X\left(S_{k}\right)\right)\right\}+E_{x}^{(n)}\left\{g\left(X\left(S_{k^{\prime}+1}\right)\right)\right\} .
$$

The fact $h \geqq c$ on $K-V$ now implies $P_{x}^{(n)}\left[X\left(S_{k}\right) \in K-V\right] \rightarrow 0$ as $k \rightarrow \infty$. Choose $k^{\prime}$ so that the second term on the right side of (5.3) is smaller than $\varepsilon$ when $x=x_{0}$. By (5.1) $\lim _{l} E_{x_{l}}^{(n)}\left\{g\left(X\left(S_{k^{\prime}+1}\right)\right)\right\}=E_{x_{0}}^{(n)}\left\{g\left(X\left(S_{k^{\prime}+1}\right)\right)\right\}$. Now write the first term on the right side of (5.2) as

$$
\begin{aligned}
\sum_{k=0}^{k^{\prime}} E_{x}^{(n)}\left\{R_{0}^{(n)} f_{1}\left(X\left(S_{k}\right)\right) ; X\left(S_{k}\right)\right. & \in V-A\}+\sum_{k=0}^{k^{\prime}} E_{x}^{(n)}\left\{h\left(X\left(S_{k}\right)\right) f_{1}\left(X\left(S_{k}\right)\right) ; X\left(S_{k}\right) \in K-V\right\} \\
& +\sum_{k=0}^{k^{\prime}} E_{x}^{(n)}\left\{E_{X\left(S_{k}\right)}^{(n)} \int_{0}^{s}\left[f_{1}\left(X_{t}\right)-f_{1}\left(X_{0}\right)\right] d t ; X\left(S_{k}\right) \in K-V\right\} .
\end{aligned}
$$

Then applying (5.2) to $x=x_{0}$ and $x_{l}, n \geqq N, f_{1}=f$, noting the facts that $\mid f\left(X_{t}\right)-$ $f\left(X_{0}\right) \mid<\varepsilon$ when $X_{0} \in K-V$ and $t<S$, that $\left\{X\left(S_{k}\right) \in V-\Delta\right\}, k=0,1, \ldots$, are disjoint, and that $\left|R_{0}^{(n)} f(y)\right| \leqq g(y)\|f\|$, and using (5.1), we have

$$
\begin{aligned}
\varlimsup_{l}\left|R_{0}^{(n)} f\left(x_{l}\right)-R_{0}^{(n)} f\left(x_{0}\right)\right| \leqq & 2\|f\| \sup _{x \in V} g(x)+2 \varepsilon\|g\| \\
& +2\|f\| E_{x_{0}}^{(n)}\left\{g\left(X\left(S_{k^{\prime}+1}\right)\right)\right\} \leqq 2 \varepsilon(\|g\|+2\|f\|) .
\end{aligned}
$$

Proposition 5.1 then implies $\varlimsup_{l}\left|R_{0}^{(\infty)} f\left(x_{l}\right)-R_{0}^{(\infty)} f\left(x_{0}\right)\right| \leqq 2 \varepsilon(\|g\|+2\|f\|)$. The proof is complete. 
Corollary 5.3. For $f \in \mathscr{C}_{0}$ and $\lambda>0,\left\{R_{\lambda}^{(n)} f, n \geqq 1\right\}$ is a Cauchy sequence in $\mathscr{M}_{0}$ and the limit $R_{\lambda}^{(\infty)} f=\lim _{n} R_{\lambda}^{(n)} f$ is in $\mathscr{C}_{0}$.

Proof. From the resolvent equation we have

$$
R_{\lambda}^{(n)} f=\sum_{k=0}^{\infty}(\mu-\lambda)^{k}\left(R_{\mu}^{(n)}\right)^{k+1} f
$$

if $|\mu-\lambda|<\|g\|^{-1} \leqq\left\|R_{\mu}^{(n)}\right\|^{-1}$. Now if $R_{\mu}^{(n)} f_{1}$ converges uniformly to a function in $\mathscr{C}_{0}$ for any $f_{1} \in \mathscr{C}_{0}$, then $\left(R_{\mu}^{(n)}\right)^{k+1} f$ does the same (consider, e.g., the case $k=1$ : we have $\left.\left(R_{\mu}^{(n)}\right)^{2} f=R_{\mu}^{(n)}\left(R_{\mu}^{(n)} f-R_{\mu}^{(\infty)} f\right)+R_{\mu}^{(n)} R_{\mu}^{(\infty)} f \rightarrow R_{\mu}^{(\infty)} R_{\mu}^{(\infty)} f\right)$; therefore $R_{\lambda}^{(n)} f$ converges uniformly to a function in $\mathscr{C}_{0}$ if $|\mu-\lambda|<\|g\|^{-1}$. Now the corollary follows from successive extension of the facts in Propositions 5.1 and 5.2.

It is immediate that each $R_{\lambda}^{(\infty)}$ is a positive operator on $C_{0},\left\|R_{\lambda}^{(\infty)}\right\| \leqq\|g\|$, $\left\|\lambda R_{\lambda}^{(\infty)}\right\| \leqq 1$ and $R_{\lambda}^{(\infty)}-R_{\mu}^{(\infty)}=(\mu-\lambda) R_{\lambda}^{(\infty)} R_{\mu}^{(\infty)}$. Before proving $\lim _{\lambda \rightarrow \infty} \lambda R_{\lambda}^{(\infty)} f=f$ uniformly for each $f \in \mathscr{C}_{0}$ we establish the following fact, to be used in Section 6.

Corollary 5.4. Let $D \in \mathscr{D}_{k}$. For $n \geqq k$ and $\lambda \geqq 0$ let $f_{n \lambda}(x)=E_{x}^{(n)}\left\{e^{-\lambda T_{D}}\right\}=$ $\hat{E}_{x}^{(n)}\left\{\prod_{\alpha<\tau_{D}}\left[e_{n}\left(Z_{\alpha}\right) /\left(\lambda+e_{n}\left(Z_{\alpha}\right)\right)\right]\right\}$ (see (4.11)). Then $f_{n \lambda}$ converges uniformly to a function in $\mathscr{C}$ as $n \rightarrow \infty$. (Note that $f_{n}=1$ on D.)

Proof. By considering the processes $X^{(n)}, n \geqq k$, stopped at the first hitting of $D$ we may assume $D=\{\Delta\}$. Now $f_{n \lambda}=1-\lambda R_{\lambda}^{(n)} f$ where $f=1_{K-A}$. But $R_{0}^{(n)} f=g$. Hence from Propositions 5.1 and 5.2 and successive application of (5.4) we see that $R_{\lambda}^{(n)} f$ converges uniformly to a function in $\mathscr{C}_{0}$, and the desired fact follows.

Proposition 5.5. For $f \in \mathscr{C}_{0}, \lambda R_{\lambda}^{(\infty)} f$ converges pointwise to $f$ as $\lambda \rightarrow \infty$.

Because of its length, the proof is given a whole section - Section 6.

Corollary 5.6. For $f \in \mathscr{C}_{0}, \lambda R_{\lambda}^{(\infty)} f$ converges uniformly to $f$ as $\lambda \rightarrow \infty$.

Proof. That this follows from Proposition 5.5 and the fact that $\left\{R_{\lambda}^{(\infty)}, \lambda>0\right\}$ is a resolvent on $\mathscr{C}_{0}$ must be well-known: one shows that the closure of the common range $R_{\lambda}\left(\mathscr{C}_{0}\right)$ is $\mathscr{C}_{0}$ using the Hahn-Banach theorem; but then for $f$ in this closure one has the desired convergence.

Now by the Hille-Yoshida theorem there exists a unique strongly continuous semigroup $\left\{P_{t}^{(\infty)}, t \geqq 0\right\}$ of positive contraction operators on $\mathscr{C}_{0}\left(P_{0}^{(\infty)}=\right.$ the identity operator) such that $\left\{R_{\lambda}^{(\infty)}, \lambda \geqq 0\right\}$ is its resolvent. Using the theorem of Blumenthal one finds a unique (up to equivalence) Hunt process $X^{(\infty)}$ on the state space $K$, with $\Delta$ as the death point, such that the $P_{t}^{(\infty)}$ are its transition operators on $\mathscr{C}_{0}$ and therefore $\left\{R_{\lambda}^{(\infty)}, \lambda \geqq 0\right\}$ is it resolvent on $\mathscr{C}_{0}$. Let

$$
R_{\lambda}^{(\infty)} f(x)=E_{x}^{(\infty)} \int_{0}^{\infty} e^{-\lambda t} f\left(X_{t}\right) d t
$$

for any $f \in \mathscr{M}$ and $\lambda>0$ (where $E_{x}^{(\infty)}$ denotes expectation with respect to $P_{x}^{(\infty)}$ ); then of course for $f \in \mathscr{C}, R_{\lambda}^{(\infty)} f \in \mathscr{C}$ and $R_{\lambda}^{(n)} f \rightarrow R_{\lambda}^{(\infty)} f$ uniformly. We may assume the sample space of $X^{(\infty)}$ is $\Omega$ and so we write $X^{(\infty)}=\left(X_{t}, P_{x}^{(\infty)}\right)$. Let us prove a uniform regularity condition for the $X^{(n)}, 1 \leqq n \leqq \infty$. Let $E(x, \delta)=\{y \mid \rho(x, y) \geqq \delta\}$. 
Proposition 5.7. Given $\varepsilon>0$ and $\delta>0$ there exists $c>0$ such that for all $x$ and $1 \leqq n \leqq \infty$

We need a lemma.

$$
P_{x}^{(n)}\left[T_{E(x, \delta)}<c\right]<\varepsilon .
$$

Lemma 5.8. Let $D \in \mathscr{D}_{n}$ and $y \in D$. For $\varepsilon_{1}>0$ and $\delta_{1}>0$ there is a neighborhood $V$ of $y$ such that $H_{D \cup E(y, \delta)}^{(n)}(x, E(y, \delta))<\varepsilon_{1}$ for all $x \in V$.

Proof. From (4.8) and (4.10) $H_{D}^{(n)}(x, \cdot)=H_{D}(x, \cdot)$ and $E_{x}^{(n)}\left\{T_{D}\right\}=g_{D}(x)$. Since if $x \rightarrow z \in D$ then $H_{D}(x, \cdot)$ converges weakly to the unit mass at $z$ and since $g_{D}(x) \rightarrow 0$ as $x \rightarrow D$ and is bounded away from 0 when $x$ is bounded away from $D$, it is a routine matter to obtain the conclusion of the lemma.

Proof of Proposition 5.7. That there exists $c>0$ such that (5.5) holds for all $x$ and $n=\infty$ is an easy consequence of Corollary 5.6. Let $n<\infty$ be fixed. Applying Lemma 5.8 to $D=\{\Delta\}$ we obtain a neighborhood $V_{\Delta}$ of $\Delta$ with diam $V_{\Delta}<\delta / 2$ such that $H_{E(\Delta, \delta / 2) \cup \Delta}^{(n)}(x, E(\Delta, \delta / 2))<\varepsilon$ for $x \in V_{A}$, so that (5.5) holds for $x \in V_{\Delta}$ and any $c$. Next for each $y \in V_{n i}-V_{\Delta}$ let $0<\delta_{1} \leqq \delta / 2$ be such that $\overline{K-E\left(y, \delta_{1}\right)} \subset U_{n i}-\Delta$, so that $\inf \left\{h_{n}(z) \mid z \in V_{n i}-E\left(y, \delta_{1}\right)\right\}>0$. Applying Lemma 5.8 to $D=\left(K-\bigcup_{j<i} U_{n j}\right) \cup \Delta$ and observing $D-E\left(y, \delta_{1}\right)=V_{n i}-E\left(y, \delta_{1}\right)$, we obtain a neighborhood $V_{y}$ of $y$ with diam $V_{y}<\delta / 2$ such that $H_{D \cup E\left(y, \delta_{1}\right)}^{(n)}\left(x, V_{n i}-E\left(y, \delta_{1}\right)\right)>1-\varepsilon / 2$ for $x \in V_{y}$. It is then clear that there exists $c=c_{y}>0$ such that (5.5) holds for all $x \in V_{y}$. That there exists $c>0$ that works for all $x$ (and this fixed $n$ ) is because $K-V_{\Delta}$ is compact. To complete the proof it sufficies to show that for any $y$ there exist $c_{0}>0, n_{0}>0$ and a neighborhood $V$ of $y$ such that $P_{x}^{(n)}\left[T_{E(y, \delta / 2)}<c_{0}\right]<\varepsilon$ for $x \in V$ and $n>n_{0}$. Suppose the contrary. Then for some $y$ there exist $n_{k} \rightarrow \infty, x_{k} \rightarrow y$ and $c_{k} \downarrow 0$ such that $P_{x_{k}}^{\left(n_{k}\right)}\left[T<c_{k}\right] \geqq \varepsilon$, where $T=T_{E(y, \delta / 2)}$. Choose $f \in \mathscr{C}$ such that $0 \leqq f \leqq 1$, $f(y)=1$ and $f=0$ on $E(y, \delta / 2)$. Fix $\lambda>0$ such that $\left\|\lambda R_{\lambda}^{(\infty)} f-f\right\|<\varepsilon / 4$. Then since $\left\|\lambda R_{\lambda}^{\left(n_{k}\right)} f-\lambda R_{\lambda}^{(\infty)} f\right\| \rightarrow 0$ as $k \rightarrow \infty$ we have $\lim _{k} \lambda R_{\lambda}^{\left(n_{k}\right)} f\left(x_{k}\right)=\lambda R_{\lambda}^{(\infty)} f(y)>1-\varepsilon / 4$.

On the other hand (assuming $\varepsilon \leqq 1$ )

$$
\begin{aligned}
\lambda R_{\lambda}^{\left(n_{k}\right)} f\left(x_{k}\right)= & E_{x}^{\left(n_{k}\right)}\left\{\int_{0}^{T} \lambda e^{-\lambda t} f\left(X_{t}\right) d t+\lambda \int_{T}^{\infty} e^{-\lambda t} f\left(X_{t}\right) d t\right\} \\
\leqq & E_{x_{k}}^{\left(n_{k}\right)}\left\{1-e^{-\lambda c_{k}} ; T<c_{k}\right\}+E_{x_{k}}^{\left(n_{k}\right)}\left\{1-e^{-\lambda T} ; T \geqq c_{k}\right\} \\
& +E_{x_{k}}^{\left(n_{k}\right)}\left\{e^{-\lambda T} \lambda R_{\lambda}^{\left(n_{k}\right)} f\left(X_{t}\right) ; T<\infty\right\} \\
\leqq & \left(1-e^{-\lambda c_{k}}\right)+(1-\varepsilon)+3 \text { rd term } .
\end{aligned}
$$

Since $X_{T} \in E(y, \delta / 2)$ if $T<\infty$ and since $\lambda R_{\lambda}^{(\infty)} f<\varepsilon / 4$ on $E(y, \delta / 2)$ we obtain

$$
\varlimsup_{k} \lambda R_{\lambda}^{\left(n_{k}\right)} f\left(x_{k}\right) \leqq 1-\varepsilon+\varepsilon / 4=1-3 \varepsilon / 4 .
$$

Thus we have the impossibility $1-\varepsilon / 4<1-3 \varepsilon / 4$.

Write $P_{t}^{(n)} f(x)=E_{x}^{(n)} f\left(X_{t}\right)$ for $f \in \mathscr{M}$. From the above proposition it is easy to see that for $f \in \mathscr{C}$, the family $\left\{P_{t}^{(n)} f(x), 1 \leqq n \leqq \infty, x \in K\right\}$ of functions on $[0, \infty)$ is equicontinuous. Hence using the convergence $R_{\lambda}^{(n)} f(x) \rightarrow R_{\lambda}^{(\infty)} f(x)$ for all $\lambda$ and the uniqueness of the Laplace transform of a bounded continuous function we easily obtain the convergence $P_{t}^{(n)} f(x) \rightarrow P_{t}^{(\infty)} f(x)$ for $x \in K, t \geqq 0$, and $f \in \mathscr{C}$. 
Proposition 5.9. The finite dimensional distributions of $X^{(n)}$ converge weakly to the corresponding ones of $X^{(\infty)}$ under any common initial distribution.

Proof. It sufficies to show

$$
E_{x}^{(n)}\left\{f_{1}\left(X_{t_{1}}\right) \ldots f_{k}\left(X_{t_{k}}\right)\right\} \rightarrow E_{x}^{(\infty)}\left\{f_{1}\left(X_{t_{1}}\right) \ldots f_{k}\left(X_{t_{k}}\right)\right\}
$$

for $x \in K, 0 \leqq t_{1}<\cdots<t_{k}$, and $f_{1}, \ldots, f_{k}$ in $\mathscr{C}$. We prove by induction. The case $k=1$ is already established. We give the induction step for $k=2$ as the general case is similar. Write $s=t_{1}, t=t_{2}-t_{1}$. Then by the induction hypothesis

$$
\begin{aligned}
& \int_{0}^{\infty} e^{-\lambda t} E_{x}^{(n)}\left\{f_{1}\left(X_{s}\right) f_{2}\left(X_{s+t}\right)\right\} d t=\int_{0}^{\infty} e^{-\lambda t} d t \int P_{x}^{(n)}\left[X_{s} \in d y\right] f_{1}(y) P_{t}^{(n)} f_{2}(y) \\
& =\int P_{x}^{(n)}\left[X_{s} \in d y\right] f_{1}(y) R_{\lambda}^{(n)} f_{2}(y) \rightarrow \int P_{x}^{(\infty)}\left[X_{s} \in d y\right] f_{1}(y) R_{\lambda}^{(\infty)} f_{2}(y) \\
& =\int_{0}^{\infty} e^{-\lambda t} d t \int P_{x}^{(\infty)}\left[X_{s} \in d y\right] f_{1}(y) P_{t}^{(\infty)} f_{2}(y)=\int_{0}^{\infty} e^{-\lambda t} E_{x}^{(\infty)}\left\{f_{1}\left(X_{s}\right) f_{2}\left(X_{s+t}\right)\right\} d t
\end{aligned}
$$

since $R_{\lambda}^{(n)} f_{2}$ converges uniformly to $R_{\lambda}^{(\infty)} f_{2}$ and the latter is in $\mathscr{C}$. Now the fact that $\left\{P_{t}^{(n)} f_{2}(y), 1 \leqq n \leqq \infty, y \in K\right\}$ is equicontinuous implies that the family $\left\{E_{x}^{(n)}\left\{f_{1}\left(X_{s}\right) f_{2}\left(X_{s+t}\right)\right\}, 1 \leqq n \leqq \infty\right\}$ of functions of $t$ is equicontinuous. By the above convergence we thus have $E_{x}^{(n)}\left\{f_{1}\left(X_{s}\right) f_{2}\left(X_{s+t}\right)\right\} \rightarrow E_{x}^{(\infty)}\left\{f_{1}\left(X_{s}\right) f_{2}\left(X_{s+t}\right)\right\}$. The proposition is proved.

\section{Pointwise Convergence of $\lambda R_{\dot{\lambda}}^{(\infty)} f$ for $f \in \mathscr{C}_{0}$}

We shall now prove Proposition 5.5, which asserts that, for $f \in \mathscr{C}_{0}, \lambda R_{\lambda}^{(\infty)} f \rightarrow f$ pointwise as $\lambda \rightarrow \infty$. To establish this convergence we shall prove the following for the generalized random walks $Z^{(n)}=\left(Z_{\alpha}, Q_{x}^{(n)}\right)$ constructed in Section 4:

$$
\lim _{\varepsilon \downarrow 0} \varlimsup_{n \rightarrow \infty} Q_{x}^{(n)}\left[\sum_{\alpha<\tau_{D}} h_{n}\left(Z_{\alpha}\right)<\varepsilon\right]=0
$$

for $D \in \mathscr{D}_{\infty}$ and $x \notin D$. Recall $\tau_{D}$ is the first hitting time of $D$ for $\left(Z_{\alpha}\right)$ and $h_{n}(x)=$ $g_{D(n, x)}(x)$ is the expected holding time at $x$ for the process $X^{(n)}$. Thus if in $X^{(n)}$ we identify sample points whose paths have the same trajectory, then $\sum_{\alpha<\tau_{D}} h_{n}\left(Z_{\alpha}\right)$ is the first hitting time of $D$, with $Z_{\alpha}$ interpreted as the $\alpha$-th jump. From (6.1) and the behavior of (possibly infinite) sums of independent exponentially distributed random variables it is not difficult to obtain the following for the processes $X^{(n)}=\left(X_{t}, P_{x}^{(n)}\right)$ :

$$
\lim _{\varepsilon \downarrow 0} \varlimsup_{n} P_{x}^{(n)}\left[T_{D}<\varepsilon\right]=0
$$

for $D \in \mathscr{D}_{\infty}$ and $x \notin D$. Using this one easily proves Proposition 5.5. However we prefer an "elementary" proof (based on (6.1)).

Proof of Proposition 5.5. We may assume $x \neq \Delta$. Let $\varepsilon_{1}>0$ and choose $D \in \mathscr{D}_{\infty}$ with $x \notin D$ and $K-D \subset\left\{y|| f(x)-f(y) \mid<\varepsilon_{1}\right\}$. Writing $T$ for $T_{D}$ we have

$$
\begin{aligned}
\left|\lambda R_{\lambda}^{(n)} f(x)-f(x)\right| & \leqq\left|E_{x}^{(n)} \int_{0}^{T} \lambda e^{-\lambda t} f\left(X_{t}\right) d t-f(x)\right|+\left|E_{x}^{(n)} \int_{T}^{\infty} \lambda e^{-\lambda t} f\left(X_{t}\right) d t\right| \\
& \leqq E_{1} E_{x}^{(n)}\left\{1-e^{-\lambda T}\right\}+|f(x)| E_{x}^{(n)}\left\{e^{-\lambda T}\right\}+E_{x}^{(n)}\left\{e^{-\lambda T}\left|\lambda R_{\lambda}^{(n)} f\left(X_{T}\right)\right|\right\} .
\end{aligned}
$$


This implies $\lim _{\lambda \rightarrow \infty} \lambda R_{\lambda}^{(\infty)} f(x)=f(x)$ by Corollary 5.3 if we can show

$$
\lim _{\lambda \rightarrow \infty} \varlimsup_{n} E_{x}^{(n)}\left\{e^{-\lambda T}\right\}=0 .
$$

Because of (4.11) it suffices to show

where $\tau=\tau_{D}$. But

$$
\lim _{\lambda \rightarrow \infty} \varlimsup_{n} \hat{E}_{x}^{(n)}\left\{\prod_{\alpha<\tau} \frac{e_{n}\left(Z_{\alpha}\right)}{\lambda+e_{n}\left(Z_{\alpha}\right)}\right\}=0
$$

$$
\log \prod_{\alpha<\tau} \frac{e_{n}\left(Z_{\alpha}\right)}{\lambda+e_{n}\left(Z_{\alpha}\right)}=-\sum_{\alpha<\tau} \log \left[\lambda h_{n}\left(Z_{\alpha}\right)+1\right] \leqq-c(\lambda) \lambda \sum_{\alpha<\tau} h_{n}\left(Z_{\alpha}\right)
$$

where $c(\lambda)=(\lambda\|g\|)^{-1} \log [\lambda\|g\|+1]$, because $a^{-1} \log (a+1)$ is a decreasing function of $a>0$ and $h_{n} \leqq\|g\|$. Hence the left side of (6.2) is dominated by

$$
\lim _{\lambda \rightarrow \infty} \varlimsup_{n} \hat{E}_{x}^{(n)}\left\{\exp \left[-c(\lambda) \lambda \sum_{\alpha<\tau} h_{n}\left(Z_{\alpha}\right)\right]\right\}
$$

which has value zero because of (6.1) and the fact $c(\lambda) \lambda \rightarrow \infty$ as $\lambda \rightarrow \infty$.

The rest of the section is devoted to the proof of (6.1). For this we need to define a stochastic process in which are imbedded all the generalized random walks $Z^{(n)}$. This is in fact the projective limit process of the sequence of processes $Z^{(n)}$ in the sense of Bochner [4]; it is entirely similar to the one defined in Dawson [5]. That this projective limit process exists is of course because there is a natural imbedding of $Z^{(n)}$ in $Z^{(k)}$ for each pair $n<k$. First recall that $\mathscr{W}=\prod_{\alpha<\pi} K_{\alpha}, K_{\alpha}=K$, is the common sample space of the $Z^{(n)}$ and the $Z_{\alpha}$ are the coordinate mappings on $\mathscr{W}$. For each $n$ let $\sigma_{n}$ be the stopping time on $\left(\mathscr{W}, Z_{\alpha}\right)$ defined by: $\sigma_{n}=\tau_{D(n, x)}$ if $Z_{0}=x$. Let $\sigma_{n \alpha}, \alpha \leqq \pi$, be the iterates of $\sigma_{n}$, i. e., $\sigma_{n 0}=0, \sigma_{n, \alpha+1}=\sigma_{n \alpha}+\sigma_{n}\left(\theta_{\sigma_{n \alpha}}\right)$, and $\sigma_{n \alpha}=\sup _{\beta<\alpha} \sigma_{n \beta}$ for a limit ordinal $\alpha$.

Definition. Let $\mathscr{W}_{n}=\left\{w_{0} \in \mathscr{W} \mid \sigma_{n \alpha}\left(w_{0}\right)=\alpha\right.$ for all $\left.\alpha<\pi\right\} ; \tilde{\mathscr{W}}_{\infty}=\prod_{n=1}^{\infty} \mathscr{W}_{n} ; \pi_{n}$ be the projection of $\tilde{\mathscr{W}}_{\infty}$ to $\mathscr{W}_{n} ; Z_{\alpha}^{(n)}=Z_{\alpha} \circ \pi_{n}$ for $\alpha \leqq \pi ; \tau^{(n)}=\tau \circ \pi_{n}$ for a stopping time on $\left(\mathscr{W}, Z_{\alpha}\right) ; Z_{\tau}^{(n)}=Z_{\tau} \circ \pi_{n}$ for such $\tau$.

Definition. Let $\mathscr{W}_{\infty}=\left\{w \in \tilde{\mathscr{W}}_{\infty} \mid Z_{\sigma_{n \alpha}}^{(n+1)}(w)=Z_{\alpha}^{(n)}(w)\right.$ for all $\left.n \geqq 1, \alpha \leqq \pi\right\} ; \mathscr{S}_{n}$ be the $\sigma$-field on $\mathscr{W}_{\infty}$ generated by $Z_{\alpha}^{(n)}, \alpha<\pi ; \mathscr{S}$ be the minimal $\sigma$-field containing all the $\mathscr{S}_{n}$.

$\pi_{n}: \mathscr{W}_{\infty} \rightarrow \mathscr{W}_{n}$ is onto. For each $x$, since $Q_{x}^{(n)}\left(\mathscr{W}_{n}\right)=1, Q_{x}^{(n)} \circ \pi_{n}^{-1}$ defines a probability measure on $\mathscr{S}_{n}$. Now clearly $\mathscr{S}_{n} \subset \mathscr{S}_{n+1}$. From the fact $Q_{y}^{(n+1)}\left[Z\left(\sigma_{n}\right) \in B\right]=$ $H_{D(n, y)}(y, B)=Q_{y}^{(n)}\left[Z_{1} \in B\right]$ and the left continuity of $Z^{(n)}$ and $Z^{(n+1)}$ one obtains by induction

$$
Q_{x}^{(n+1)}\left[Z\left(\sigma_{n \alpha_{i}}\right) \in B_{i}, 1 \leqq i \leqq j\right]=Q_{x}^{(n)}\left[Z\left(\alpha_{i}\right) \in B_{i}, 1 \leqq i \leqq j\right]
$$

thus $Q_{x}^{(n+1)} \circ \pi_{n+1}^{-1}=Q_{x}^{(n)} \circ \pi_{n}^{-1}$ on $\mathscr{S}_{n}$. Using the Kolmogorov extension theorem we obtain probability measures $Q^{x}$ on $\left(\mathscr{W}_{\infty}, \mathscr{S}\right)$ such that

$$
Q^{x}(\Lambda)=Q_{x}^{(n)}\left(\pi_{n}^{-1}(\Lambda)\right), \quad \Lambda \in \mathscr{P}_{n}
$$


The triple $Z^{(\infty)}=\left(\mathscr{W}_{\infty}, Z_{\alpha}^{(n)}, Q^{x}\right)$, where $n \geqq 1, \alpha \leqq \pi, x \in K$, is the projective limit process of the sequence $Z^{(n)}$. With a fixed $n\left(\mathscr{W}_{\infty}, Z_{\alpha}^{(n)}, Q^{x}\right)$ is a process equivalent to $Z^{(n)}$. The Markov property of the $Z^{(n)}$ gives rise to a Markov property of $Z^{(\infty)}$; to state it we need to introduce some $\sigma$-fields.

Definition. For a stopping time on $\left(\mathscr{W}, Z_{\alpha}\right)$, let $\mathscr{F}_{\tau}^{(n)}$ be the $\sigma$-field (on $\mathscr{W}_{\infty}$ ) generated by sets of the form $\left\{Z_{\alpha}^{(n)} \in B, \alpha \leqq \tau^{(n)}\right\} ; \mathscr{G}_{\tau}^{(n)}$ be the $\sigma$-field generated by sets of the form $\left\{Z_{\alpha}^{(k)} \in B, \alpha \leqq \sigma_{n, \tau^{(n)}}^{(k)}\right\}, k \geqq n$, where $\sigma_{n, \tau^{(n)}}^{(k)}(w)=\sigma_{n, \tau^{(n)}(w)}^{(k)}(w)$.

Observe that for $\tau=\tau_{D}$ with $D \in \mathscr{D}_{m}$ or $\tau=\sigma_{m \alpha}$, where $m \leqq n$, or for other similar stopping times $\tau, \mathscr{F}_{\tau}^{(n)} \subset \mathscr{\mathscr { F }}_{\tau}^{(k)}$ for $k>n$ and $\mathscr{G}_{\tau}^{(n)}$ is the minimal $\sigma$-field containing all $\mathscr{F}_{\tau}^{(k)}$ with $k \geqq n$.

Definition. The shift operators $\theta_{n \alpha}, \alpha \leqq \pi, n \geqq 1$, on $\mathscr{W}_{\infty}$ are defined by requiring $Z_{\beta}^{(k)}\left(\theta_{n \alpha} w\right)=Z_{\sigma_{n \alpha+\beta}}^{(k)}(w)$ for all $k \geqq n, \beta<\pi$.

It is easily seen that $\theta_{n \alpha}$ is well-defined: for each $w \in \mathscr{W}_{\infty}$ there is a unique $\theta_{n \alpha} w$ with the required property. The Markov property of $Z^{(\infty)}$ can now be stated as follows: for a stopping time $\tau$ on $\left(\mathscr{W}, Z_{\alpha}\right)$ and a bounded real $\mathscr{S}$-measurable $\eta$

$$
E^{x}\left\{\eta\left(\theta_{n, \tau^{(n)}}\right) \mid \mathscr{G}_{\tau}^{(n)}\right\}=E^{Z_{\tau}^{(n)}}\{\eta\}
$$

for any $x$, where $E^{x}$ stands for expectation with respect to $Q^{x}$. This is easily proved from the (strong) Markov property of the $Z^{(n)}$. In order to facilitate understanding of what we do we shall interprete things in terms of the to-be-constructed process. $Z^{(\infty)}$ is a process that provides all the information about the trajectories of the to-be-constructed process. Thus stopping times of the latter defined from hitting times have a meaning in $Z^{(\infty)}$, and (6.3) states the strong Markov property at certain such times.

We can now begin the proof of (6.1). Thus we assume $D$ is a fixed set in $\mathscr{D}_{\infty}$ and $x$ is a fixed point in $K-D$. Without loss of generality we may assume $D \in \mathscr{D}_{1}$; hence $D \subset D(n, y)$ for $y \notin D$ and $n \geqq 1$.

Definition. Writing $\tau$ for $\tau_{D}$, we define functions $\xi_{n}$, $\xi$ on $\mathscr{W}_{\infty}$ and $u$ on $K$ as follows:

$$
\xi_{n}=\sum_{\alpha<\tau^{(n)}} h_{n}\left(Z_{\alpha}^{(n)}\right) ; \quad \xi=\underline{\lim } \xi_{n} ; \quad u(y)=Q^{y}[\xi=0] .
$$

Note that if $y \in D$, then $\tau^{(n)}=0$ a.s. $Q^{y}$ for all $n$; hence $u(y)=1$. From the next proposition $\xi_{n} \rightarrow \xi$ a.s. $Q^{y}$ for any $y$. $\xi$ is the hitting time of $D$ for the to-be-constructed process if sample points whose paths have the same trajectory are identified.

Proposition 6.1. (i) $E^{y}\left\{\xi_{n}\right\}=g_{D}(y)$; (ii) $\left\{\xi_{n}, \mathscr{F}_{\tau}^{(n)}, n \geqq 1\right\}$ is a uniformly integrable martingale with respect to $Q^{y}$, for any $y$.

Proof. $E^{y}\left\{\xi_{n}\right\}=\hat{E}_{y}^{(n)}\left\{\sum_{\alpha<\tau} h_{n}\left(Z_{\alpha}\right)\right\}=g_{D}(y)$ by (4.10) and the definition of $Q^{y}$. For (ii) we have observed $\mathscr{F}_{\tau}^{(n)} \subset \mathscr{F}_{\tau}^{(n+1)}$. To prove the martingale equality it suffices to show $\hat{E}_{y}^{(n)}\left\{\sum_{\alpha<\tau} h_{n}\left(Z_{\alpha}\right) ; A\right\}=\hat{E}_{y}^{(n+1)}\left\{\sum_{\alpha<\tau} h_{n+1}\left(Z_{\alpha}\right) ; \Lambda^{\prime}\right\}$ where $\Lambda=\left\{Z\left(\alpha_{i}\right) \in B_{i}\right.$, $\left.\alpha_{i} \leqq \tau, 1 \leqq i \leqq j\right\}$ and $\Lambda^{\prime}=\left\{Z\left(\sigma_{n \alpha_{i}}\right) \in B_{i}, \sigma_{n \alpha_{i}} \leqq \tau, 1 \leqq i \leqq j\right\}$. But this is easily deduced from the fact $\hat{E}_{z}^{(n+1)}\left\{\sum_{\alpha<\sigma_{n}} h_{n+1}\left(Z_{\alpha}\right)\right\}=h_{n}(z)$ (see (4.10)). It remains to show the uniform integrability. For arbitrary $n$ and $a>0$ let $\tau_{a}=\inf \left\{\alpha \leqq \tau \mid \sum_{\beta<\alpha} h_{n}\left(Z_{\beta}\right) \geqq a\right\}$ if 

there is such $\alpha$, and $=\tau$ otherwise. Since $h_{n} \leqq g$ we have $\sum_{\alpha<\tau_{a}^{(n)}} h_{n}\left(Z_{\alpha}^{(n)}\right) \leqq a+\|g\|$.
Hence if $b>a+\|g\|$

$$
\begin{aligned}
E^{y}\left\{\xi_{n}-a ; \xi_{n}>b\right\} & \leqq E^{y}\left\{\xi_{n}-a ; \tau_{a}^{(n)}<\tau^{(n)}\right\} \\
& =E^{y}\left\{\sum_{\alpha<\tau_{a}^{(n)}} h_{n}\left(Z_{\alpha}^{(n)}\right)-a+E^{Z_{a}^{(n)}}\left[\xi_{n}\right] ; \tau_{a}^{(n)}<\tau^{(n)}\right\} \\
& \leqq E^{y}\left\{\|g\|+g_{D}\left(Z_{\tau_{a}}^{(n)}\right) ; \tau_{a}^{(n)}<\tau^{(n)}\right\} \\
& \leqq 2\|g\| Q^{y}\left[\xi_{n} \geqq a\right] .
\end{aligned}
$$

Now if $a^{2}>a+\|g\|$, then using (i) we have

$$
\begin{aligned}
E^{y}\left\{\xi_{n} ; \xi_{n}>a^{2}\right\} & =E^{y}\left\{\xi_{n}-a ; \xi>a^{2}\right\}+a Q^{y}\left[\xi_{n}>a^{2}\right] \\
& \leqq 2\|g\| \cdot \frac{g_{D}(y)}{a}+a \cdot \frac{g_{D}(y)}{a^{2}}
\end{aligned}
$$

and the uniform integrability of $\left\{\xi_{n}\right\}$ with respect to $Q^{y}$ follows.

It follows from the above proposition that $\xi_{n} \rightarrow \xi$ a.s. $Q^{y}$ for every $y$. Hence (i) of the following proposition is immediate from the definition of $Q^{y}$.

Proposition 6.2. (i) $u(y)=\lim _{\varepsilon \downarrow 0} \varlimsup_{n} Q_{y}^{(n)}\left[\sum_{\alpha<\tau} h_{n}\left(Z_{\alpha}\right)<\varepsilon\right]$; (ii) $u$ is upper semicontinuous; (iii) for $D^{\prime} \in \mathscr{D}_{\infty}$ with $D \subset D^{\prime}, H_{D^{\prime}} u \geqq u$.

Proof. From Corollary 5.4 the functions $f_{n \lambda}(y)=\hat{E}_{y}^{(n)}\left\{\prod_{\alpha<\tau} \frac{e_{n}\left(Z_{\alpha}\right)}{\lambda+e_{n}\left(Z_{\alpha}\right)}\right\}$ converge (uniformly) to a continuous function $f_{\lambda}(y)$ as $n \rightarrow \infty$. Now

$$
f_{n \lambda}(y)=E^{y}\left\{\exp \left(-\sum_{\alpha<\tau^{(n)}} \log \left[\lambda h_{n}\left(Z_{\alpha}^{(n)}\right)+1\right]\right)\right\} .
$$

For $c=c(\lambda)=(\lambda\|g\|)^{-1} \log \left[\lambda h_{n}\left(Z_{\alpha}^{(n)}\right)+1\right]$ we have

$$
\begin{aligned}
c \lambda \xi_{n} & =c \lambda \sum_{\alpha<\tau^{(n)}} h_{n}\left(Z_{\alpha}^{(n)}\right) \leqq \sum_{\alpha<\tau^{(n)}} \log \left[\lambda h_{n}\left(Z_{\alpha}^{(n)}\right)+1\right] \\
& \leqq \lambda \sum_{\alpha<\tau^{(n)}} h_{n}\left(Z_{\alpha}^{(n)}\right)=\lambda \xi_{n}
\end{aligned}
$$

(as noted in the proof of Proposition $5.5 a^{-1} \log (a+1)$ is decreasing for $a>0$, and it is bounded above by 1 ). Now as $n \rightarrow \infty$, the first and last expressions above converge respectively to $c \lambda \xi$ and $\lambda \xi$ a.s. $Q^{y}$. Hence for any $y, E^{y}\{\exp (-c \lambda \xi)\} \geqq$ $f_{\lambda}(y) \geqq E^{y}\{\exp (-\lambda \xi)\}$. Let $\lambda_{n} \rightarrow \infty$ be such that $\lambda_{n} \leqq\|g\|^{-1} \log \left(\lambda_{n+1}\|g\|+1\right)=$ $c\left(\lambda_{n+1}\right) \lambda_{n+1}$. Then $f_{\lambda_{n}} \geqq f_{\lambda_{n+1}}$ and it follows that $\lim f_{\lambda_{n}}$ is upper semi-continuous. But clearly $\lim _{n} f_{\lambda_{n}}(y)=\lim _{n} E^{y}\left\{\exp \left(-\lambda_{n} \xi\right)\right\}=Q^{y}[\xi=0]=u(y)$. It remains to prove (iii). Let $D^{\prime} \in \mathscr{n}_{n}$. Clearly $\stackrel{n}{\xi} \geqq \xi\left(\theta_{n, \tau_{D}^{(n)}}\right)$. Hence by the Markov property (6.3) and (4.8)

$$
u(y)=Q^{y}[\xi=0] \leqq \hat{E}^{y}\left\{Q^{Z_{\tau^{\prime}}^{(n)}}[\xi=0]\right\}=\int H_{D^{\prime}}(y, d z) u(z) .
$$

In view of (i) above (6.1) is the same as $u(x)=0$. We now assume

$$
u(x)>0
$$

and shall prove that it contradicts hypothesis 7). Because of (ii) of the above proposition, the set $A=\{y \mid u(y) \geqq u(x) / 2\}$ is closed. We have $x \in A$ and $D \subset A$ since 
$u=1$ on $D$. Let $A_{0}=A-D$. Let $D_{n}$ be the largest set in $\mathscr{D}_{n}$ disjoint from $A_{0}$, i.e., $D_{n}=\left(K-U_{n}\right) \cup \Delta$ where $U_{n}=\bigcup\left\{U_{n i} \mid V_{n i} \cap A_{0} \neq \emptyset\right\}$. Then $D \subset D_{1} \subset \cdots \subset D_{n} \uparrow K-A_{0}$ since $\operatorname{diam} U_{n i}<1 / n$.

Proposition 6.3. There exists $\varepsilon>0$ such that $H_{D_{n}}(x, D)>\varepsilon$ for all $n$.

Proof. By (iii) of Proposition 6.2 and the fact $u=1$ on $D$ and $u<u(x) / 2$ on $D_{n}-D$

$$
\begin{aligned}
u(x) & \leqq \int H_{D_{n}}(x, d y) u(y) \leqq \frac{u(x)}{2} H_{D_{n}}(x, K-D)+H_{D_{n}}(x, D) \\
& =\frac{u(x)}{2}+\left(1-\frac{u(x)}{2}\right) H_{D_{n}}(x, D) .
\end{aligned}
$$

The proposition follows with $\varepsilon=u(x) /(3-u(x))$, which is positive by (6.4).

The proof of the next proposition will take the rest of the section.

Proposition 6.4. $g_{D_{n}}(x) \downarrow 0$.

As soon as this proposition is established, the proof of $(6.1)$ will be complete. For Propositions 6.3 and 6.4 together violate hypothesis 7) with $U=K-D$ and thus show the absurdity of the assumption (6.4). In terms of the to-be-constructed process, that (6.1) implies Proposition 6.4 means that if the process starting at $x$ hits $D$ immediately with positive probability, then it leaves $A_{0}$ immediately with probability one.

Definition. For $n \geqq 1$ and $\alpha<\pi$ let $\bar{\sigma}_{n \alpha}$ be the minimum of $\sigma_{n \alpha}$ and $\tau$ and define for $k \geqq n$

$$
\xi(n, \alpha, k)=\sum_{\alpha<\tilde{\sigma}_{n \alpha}^{(k)}} h_{k}\left(Z_{\alpha}^{(k)}\right) ; \quad \xi(n, \alpha)=\frac{\lim }{k} \xi(n, \alpha, k) .
$$

Just as in Proposition 6.1 one shows that $\{\xi(n, \alpha, k), k \geqq n\}$ is a martingale with respect to any $Q^{y}$, and it is uniformly integrable since $\xi(n, \alpha, k) \leqq \xi_{k}$. Hence $\xi(n, \alpha, k) \rightarrow \xi(n, \alpha)$ a.s. $Q^{y}$ for any $y$. In terms of the to-be-constructed process (with the aforementioned identifying of sample points), $\xi(n, \alpha)$ is the minimum of the hitting time of $D$ and the $\alpha$-th iterate of the time $T_{n}$ that equals the hitting time $D(n, y)$ when the starting point is $y$. Note that $\xi(n, \alpha)+\xi(n, \beta)\left(\theta_{n \alpha}\right)=\xi(n, \alpha+\beta)$ a.s. $Q^{y}$.

Definition. Let $\tau_{n}=\tau_{D_{n}}$ and set

$$
\begin{aligned}
& \zeta_{n k}=\xi\left(n, \tau_{n}^{(n)}, k\right)=\sum_{\alpha<\tau_{n}^{(k)}} h_{k}\left(Z_{\alpha}^{(k)}\right), \quad k \geqq n, \\
& \zeta_{n}=\underline{\lim _{k}} \zeta_{n k}=\xi\left(n, \tau_{n}^{(n)}\right) .
\end{aligned}
$$

For the same reason as in Proposition $6.1\left\{\zeta_{n k}, k \geqq n\right\}$ is a uniformly integrable martingale with respect to any $Q^{y}$ and $g_{D_{n}}(y)=E^{y}\left\{\zeta_{n k}\right\}=E^{y}\left\{\zeta_{n}\right\}\left(\zeta_{n}\right.$ is of course the hitting time of $D_{n}$ for the to-be-constructed process). Hence Proposition 6.4 will follow if we show $\zeta_{n} \downarrow 0$ a.s. $Q^{x}$ (that $\zeta_{n}$ decreases is of course trivial).

Definition. Let

$$
\begin{aligned}
\gamma_{n} & =\inf \left\{\alpha \leqq \tau_{n}^{(n)} \mid \xi(n, \alpha)>0\right\} \quad \text { if there is such } \alpha \\
& =\tau_{n}^{(n)} \quad \text { otherwise } \\
\eta_{n} & =\xi\left(n, \gamma_{n}\right) ; \quad \eta=\lim _{n} \eta_{n} .
\end{aligned}
$$


Of course $\eta_{n}$ decreases, so that its limit exists. It will be shown that $\eta=0$ a.s. $Q^{y}$ for any $y$. Thus $\eta_{n} \downarrow 0$ a.s. $Q^{y}$. Then we show that a.s. $Q^{x}, Z_{\gamma_{n}}^{(n)} \notin A_{0}$ for infinitely many $n$. But (as will be explained) if $Z_{\gamma_{n}}^{(n)}(w) \in D_{m}$ for some $m \geqq n$ then $\eta_{n}(w) \geqq \zeta_{m}(w)$. Hence we must have $\zeta_{n} \downarrow 0$ a.s. $Q^{x}$. To prove $Q^{y}[\eta=0]=1$ for any $y$ we need the following lemmas. We shall call a point $y$ instantaneous if the infimum of $g_{F}(y)$ over $F \in \mathscr{D}$ not containing $y$ is zero. By the fact $g_{F} \downarrow$ if $F \uparrow$ (see (2.2)), $y$ is instantaneous if and only if $g_{D(n, y)}(y) \downarrow 0$.

Lemma 6.5. (i) Every $y \in A_{0}$ is instantaneous; (ii) if $y$ is instantaneous, then $\sup \left\{h_{n}(z) \mid z \notin D(n, y)\right\} \leqq \sup g_{D(n, y)} \downarrow 0$.

Proof. If $y \in A_{0}$, then $h_{n}\left(Z_{0}\right) \leqq \xi_{n} \rightarrow 0$ with positive $Q^{y}$-probability. Since $Z_{0}=y$ a.s. $Q^{y}$ we must have $g_{D(n, y)}(y)=h_{n}(y) \downarrow 0$. Thus (i) is proved. The inequality in (ii) is because for $z \notin D(n, y), D(n, y) \subset D(n, z)$ and hence $h_{n}(z)=g_{D(n, z)}(z) \leqq$ $g_{D(n, y)}(z)$. If $y$ is instantaneous, then for a given $\varepsilon>0$ there is $k$ such that $g_{D(k, y)}(y)<\varepsilon$. By the continuity of $g_{D(k, y)}$ there exists $n \geqq k$ with $K-D(n, y) \subset\left\{g_{D(k, y)}<\varepsilon\right\}$. Now $g_{D(n, y)}<\varepsilon$.

Lemma 6.6. $\sup \left\{h_{n}(y) \mid y \notin D_{n}\right\} \downarrow 0$.

Proof. Recall $D_{n}=\left(K-U_{n}\right) \cup \Delta$ where $U_{n}=\bigcup\left\{U_{n i} \mid V_{n i} \cap A_{0} \neq \emptyset\right\}$. Let $\varepsilon>0$. There is a neighborhood $V$ of $D$ such that $g_{D}<\varepsilon$ on $V$. We may assume $V$ is the union of some of the $U_{k i}$ for a fixed $k$. Now by Lemma 6.5 and the compactness of $A_{0}-V$ there exists $n \geqq k$ such that $h_{n}(y)<\varepsilon$ for $y \in U_{n}^{\prime}=\bigcup\left\{U_{n i} \mid V_{n i} \cap\left(A_{0}-V\right) \neq \emptyset\right\}$. On the other hand, if $y \in U_{n}-U_{n}^{\prime}$ then $y \in U_{n i}$ for some $i$ such that $V_{n i} \cap V \neq \emptyset$; since $U_{n i} \subset V$ we have $h_{n}(y) \leqq g_{D}(y)<\varepsilon$. Hence $\sup \left\{h_{n}(y) \mid y \notin D_{n}\right\} \leqq \varepsilon$.

Lemma 6.7. $Q^{y}\left[\zeta_{n}>0\right.$ and $\gamma_{n}$ is a limit ordinal $]=0$ for all $y$ and $n$.

Proof. Let $\alpha_{m} \uparrow \alpha$. Then since $\{\xi(n, \beta, k), k \geqq n\}$ is a uniformly integrable martingale with respect to $Q^{y}$

$$
E^{y}\left\{\xi\left(n, \alpha_{m}\right)\right\}=E^{y}\left\{\xi\left(n, \alpha_{m}, n\right)\right\} \uparrow E^{y}\{\xi(n, \alpha, n)\}=E^{y}\{\xi(n, \alpha)\} .
$$

Since $\xi(n, \beta)$ increases with $\beta$ we have $\xi\left(n, \alpha_{m}\right) \uparrow \xi(n, \alpha)$ a.s. $Q^{y}$. The lemma follows.

Proposition 6.8. $Q^{y}[\eta=0]=1$ for all $y$.

Proof. Let $\varepsilon>0$ and $\delta>0$. For a fixed positive integer $N$ define stopping times $\lambda_{n}, n \geqq N$, by setting $\lambda_{n}=\inf \left\{\alpha \leqq \tau_{N}^{(n)} \mid \xi(n, \alpha, n) \geqq \delta\right\}$ if there is such $\alpha$, and $=\tau_{N}^{(n)}$ otherwise. Let $\gamma_{N}^{\prime}=$ predecessor of $\gamma_{N}$ if it has one, and $=\gamma_{N}$ otherwise. Then by Lemma $6.7 \xi\left(N, \gamma_{N}^{\prime}\right)=0$ a.s. $Q^{y}$. Now $\xi\left(m, \sigma_{N, \gamma_{N}^{\prime}}^{(m)}\right)=\xi\left(N, \gamma_{N}^{\prime}\right)$ for $m \geqq N$. Since $\xi(m, \alpha, n) \rightarrow \xi(m, \alpha)$ a.s. $Q^{y}$ and since there are only countably many pairs $(m, \alpha)$, we have

$$
Q^{y}\left[\lambda_{n}<\sigma_{N, \gamma_{N}^{\prime}}^{(n)}\right] \rightarrow 0 \quad \text { as } n \rightarrow \infty .
$$

Now let $N$ be so large that $\sup \left\{h_{N}(z) \mid z \notin D_{N}\right\}<\varepsilon \delta$ (Lemma 6.6). Define for $n \geqq N$ : $\lambda_{n}^{\prime}=\lambda_{n}+\lambda_{n}\left(\theta_{n, \lambda_{n}}\right)$ if $\lambda_{n}<\tau_{N}^{(n)}$ and $=\tau_{N}^{(n)}$ otherwise. Then since $h_{n}$ decreases we clearly have

$$
\xi\left(n, \lambda_{n}^{\prime}, n\right) \leqq 2(\delta+\varepsilon \delta) .
$$


On the other hand, for $n \geqq N$

hence

$$
\begin{aligned}
\varepsilon \delta & >E^{y}\left\{h_{N}\left(Z_{\lambda_{n}}^{(n)}\right) ; \lambda_{n}<\tau_{N}^{(n)}\right\} \\
& =E^{y}\left\{E^{Z_{\lambda_{n}}^{(n)}}\left[\sum_{\alpha<\sigma_{N}^{(n)}} h_{n}\left(Z_{\alpha}^{(n)}\right)\right] ; \lambda_{n}<\tau_{N}^{(n)}\right\} \\
& =E^{y}\left\{\sum_{\alpha<\sigma_{N}^{(n)}} h_{n}\left(Z_{\alpha}^{(n)} \circ \theta_{n, \lambda_{n}}\right) ; \lambda_{n}<\tau_{N}^{(n)}\right\} ;
\end{aligned}
$$

$$
\begin{aligned}
\varepsilon & >Q^{y}\left[\lambda_{n}<\tau_{N}^{(n)}, \sum_{\alpha<\sigma_{N}^{(n)}} h_{n}\left(Z_{\alpha}^{(n)} \circ \theta_{n, \lambda_{n}}\right) \geqq \delta\right] \\
& =Q^{y}\left[\lambda_{n}<\tau_{N}^{(n)}, \lambda_{n}+\sigma_{N}^{(n)} \circ \theta_{n, \lambda_{n}} \geqq \lambda_{n}^{\prime}\right] \\
& \geqq Q^{y}\left[\sigma_{N, \gamma_{N}}^{(n)} \leqq \lambda_{n}<\tau_{N}^{(n)}, \lambda_{n}+\sigma_{N}^{(n)} \circ \theta_{n, \lambda_{n}} \geqq \lambda_{n}^{\prime}\right] \\
& \geqq Q^{y}\left[\sigma_{N, \gamma_{N}}^{(n)} \leqq \lambda_{n}<\tau_{N}^{(n)}, \sigma_{N, \gamma_{N}}^{(n)} \geqq \lambda_{n}^{\prime}\right] .
\end{aligned}
$$

The last inequality is because that, if $\sigma_{N, \tau_{N}^{\prime}}^{(n)} \leqq \lambda_{n}<\sigma_{N, \gamma_{N}}^{(n)}$, then $\lambda_{n}+\sigma_{N}^{(n)} \circ \theta_{n, \lambda_{n}} \leqq$ $\sigma_{N, \gamma_{N}}^{(n)}$. This follows in turn from the following: $\left(U_{n}, V_{n}\right) \subset\left(U_{N}, V_{N}\right)$, so that if $Z_{\lambda_{n}}^{(n)} \in V_{N i}$, then for $\lambda_{n} \leqq \alpha<\sigma_{N}^{(n)} \circ \theta_{n, \lambda_{n}}$ we have $Z_{\alpha}^{(n)} \in U_{N i}$. Now by (6.5) we can choose $n_{1} \geqq N$ so large that $Q^{y}\left[\lambda_{n}<\sigma_{N, \gamma_{N}^{\prime}}^{(n)}\right]<\varepsilon$ for $n \geqq n_{1}$. From this and (6.7) we obtain $Q^{y}\left[\lambda_{n}^{\prime} \leqq \sigma_{N, \gamma_{N}}^{(n)}\right]<2 \varepsilon$ for $n \geqq n_{1}$. This together with (6.6) yields

$$
\begin{aligned}
Q^{y}\left[\eta_{N}>2(\delta+\varepsilon \delta)\right] & =Q^{y}\left[\varlimsup_{n} \xi\left(n, \sigma_{N, \gamma_{N}}^{(n)}, n\right)>2(\delta+\varepsilon \delta)\right] \\
& \leqq Q^{y}\left[\varlimsup_{n} \xi\left(n, \lambda_{n}^{\prime}, n\right)>2(\delta+\varepsilon \delta)\right]+2 \varepsilon=2 \varepsilon .
\end{aligned}
$$

The proposition now follows from the arbitrariness of $\delta$ and $\varepsilon$. Let $\eta_{n}^{\prime}=\eta_{n}-\eta_{n+1}$. From the above proposition $\eta_{n}=\sum_{k=n}^{\infty} \eta_{k}^{\prime}$ a.s. $Q^{y}$. Observe that
for $k<n, \eta_{k}^{\prime} \leqq \xi-\eta_{n}=\xi\left(\theta_{n, \gamma_{n}}\right)$ a.s. $Q^{y}$ for any $y$.

Proposition 6.9. $Q^{x}\left[Z_{\gamma_{n}}^{(n)} \in A_{0}\right.$ for all $\left.n \geqq N\right]=0$ for $N \geqq 1$.

Proof. Suppose the probability is positive for some $N$. Then since $Z_{\gamma_{n}}^{(n)}$ is in $D_{n}$ and thus not in $A_{0}$ when $\eta_{n}=0$, there exist $N=n_{0}<n_{1}<\cdots<n_{k}<\cdots$ such that $Q^{x}\left(\Lambda_{0}\right)$ is positive, with $\Lambda_{m}, m \geq 0$, defined as follows:

$$
A_{m}=\left\{Z_{\gamma\left(n_{k}\right)}^{\left(n_{k}\right)} \in A_{0} \text { and } \sum_{n_{k} \leqq n<n_{k+1}} \eta_{n}^{\prime}>0 \text { for all } k \geqq m\right\}
$$

where we have written $\gamma_{n_{k}}$ as $\gamma\left(n_{k}\right)$. By the remark preceeding the proposition, the Markov property of $Z^{(\infty)}$ applied at the times $\gamma\left(n_{k}\right)$ (an extension of (6.3)), and the fact $u \geqq u(x) / 2$ on $A_{0}$

$$
\begin{aligned}
Q^{x}\left(\Lambda_{0}\right) & \leqq E^{x}\left\{Q^{z_{\gamma\left(n_{1}\right)}^{\left(n_{1}\right)}}[\xi>0] ; \Lambda_{1}\right\}=E^{x}\left\{1-u\left(Z_{\gamma\left(n_{1}\right)}^{\left(n_{1}\right)}\right) ; \Lambda_{1}\right\} \\
& \leqq(1-u(x) / 2) Q^{x}\left(\Lambda_{1}\right) \leqq(1-u(x) / 2) E^{x}\left\{Q^{z_{\gamma\left(n_{2}\right)}^{\left(n_{2}\right)}}[\xi>0] ; \Lambda_{2}\right\} \\
& \leqq \cdots \leqq(1-u(x) / 2)^{m} Q^{x}\left(\Lambda_{m}\right)
\end{aligned}
$$

for any $m$. Since $u(x)>0$ we have a contradiction. 
Now we can complete the proof of Proposition 6.4 and thus that of (6.1) by showing $\zeta_{n} \downarrow 0$ a.s. $Q^{x}$. If $z=Z_{\gamma_{n}}^{(n)}(w) \notin A_{0}$, then $z \in D_{m}$ for some $m \geqq n$ and so $\sigma_{n, \gamma_{n}}^{(m)}(w) \geqq$ $\tau_{m}^{(m)}(w)$, which implies

$$
\eta_{n}(w)=\xi\left(n, \gamma_{n}\right)(w) \geqq \xi\left(m, \tau_{m}^{(m)}\right)(w)=\zeta_{m}(w) .
$$

Thus, since $\eta_{n} \downarrow 0$ a.s. $Q^{x}$ (Proposition 6.8), Proposition 6.9 implies $\zeta_{n} \downarrow 0$ a.s. $Q^{x}$.

\section{Hitting Distributions and the Expected Lifetime of $X^{(\infty)}$}

We shall now prove that the process $X^{(\infty)}=\left(X_{t}, P_{x}^{(\infty)}\right)$ found in Section 5 is the desired process.

Proposition 7.1. $E_{x}^{(\infty)}\left\{T_{A}\right\}=g(x)$ for every $x$.

Proof. Choose $D_{k} \in \mathscr{D}_{\infty}$ such that $\Delta$ is in the interior of $D_{k}$ and $D_{k} \downarrow A$. Let $f_{k} \in \mathscr{C}_{0}$ satisfy $0 \leqq f_{k} \leqq 1$ and $f_{k}=1$ on $K-D_{k}$. Then for a fixed $k$ and all sufficiently large $n$

$$
g_{D_{k}}(x)=E_{x}^{(n)}\left\{T_{D_{k}}\right\} \leqq R_{0}^{(n)} f_{k}(x) \leqq E_{x}^{(n)}\left\{T_{\Delta}\right\}=g(x)
$$

from (4.9) and (4.10). Letting $n \rightarrow \infty$ we obtain $g_{D_{k}}(x) \leqq R_{0}^{(\infty)} f_{k}(x) \leqq g(x)$. Since $g(x)-g_{D_{k}}(x)=H_{D_{k}} g(x) \leqq \max _{y \in D_{k}} g(y) \rightarrow 0$ as $k \rightarrow \infty$, we must have $\lim _{k} R_{0}^{(\infty)} f_{k}(x)=g(x)$. The proposition follows since the limit is $E_{x}^{(\infty)}\left\{T_{A}\right\}$.

Next we show that $H_{D}^{(\infty)}(x, \cdot)=H_{D}(x, \cdot)$ for all $x \in K$ and $D \in \mathscr{D}$, where $H_{D}^{(\infty)}(x, B)=P_{x}^{(\infty)}\left[X\left(T_{D}\right) \in B\right]$. As in [9] we shall make use of Theorem 3.1 of Skorokhod [13] on the family of processes $X^{(n)}, 1 \leqq n \leqq \infty$. Note that they have been defined on the same sample space $\Omega$ and with the same random variables $X_{t}$. Skorokhod's theorem is stated for the case where the time set of the processes in question is $[0,1]$. However Stone's method in [14] can be used to extend it to cover our case. Now from Proposition 5.7 the processes $X^{(n)}, 1 \leqq n \leqq \infty$, satisfy the following uniform regularity condition: for any $\delta>0$

$$
\lim _{c \downarrow 0} \sup _{1 \leqq n \leqq \infty} \sup _{0 \leqq t<c} \sup _{x \in K} P_{x}^{(n)}\left[X_{t} \in E(x, \delta)\right]=0 .
$$

This condition is sufficient for applying Skorokhod's theorem, which gives the following: if the finite dimensional distributions of $X^{(n)}$ converge weakly to the corresponding ones of $X^{(\infty)}$ under the common initial distribution $\varepsilon_{x}$ (the unit mass at $x$ ), then for any bounded real function $\phi$ on $\Omega$ that is $J_{1}$-continuous except on a subset of $P_{x}^{(\infty)}$-measure zero, we have

$$
E_{x}^{(n)}\{\phi\} \rightarrow E_{x}^{(\infty)}\{\phi\}
$$

The $J_{1}$-topology on $\Omega$ is defined as follows: $\omega_{n}$ converges to $\omega$ in the $J_{1}$-topology if and only if there exist continuous one-to-one functions $o_{n}:[0, \infty) \rightarrow[0, \infty)$ such that for each $s>0$

$$
\sup _{0 \leqq t \leqq s}\left|o_{n}(t)-t\right| \rightarrow 0 \text { and } \sup _{0 \leqq t \leqq s} \rho\left(\omega_{n}(t), \omega\left(o_{n}(t)\right)\right) \rightarrow 0
$$

as $n \rightarrow \infty$. We can apply Skorokhod's theorem since the required weak convergence of finite dimensional distributions holds from Proposition 5.9. 
Let $D \in \mathscr{D}_{N}$ where $N$ is any positive integer and let $x$ be fixed. We shall show $H_{D}^{(\infty)}(x, \cdot)=H_{D}(x, \cdot)$. Let $U_{r}=\{y \mid \rho(y, D)<r\}$ for a positive $r$, where $\rho(y, D)=$ $\inf \{\rho(y, z) \mid z \in D\}$.

Lemma 7.2. For $s>0$ there is $r, 0<r<s$, such that $P_{x}^{(\infty)}\left[T_{\bar{U}_{r}}<T_{U_{r}}\right]=0$.

Proof. If not, then one easily obtains $E_{x}^{(\infty)}\left\{T_{A}\right\}=\infty$, contradicting Proposition 7.1.

Lemma 7.3. Given $f \in \mathscr{C}$ and $\varepsilon>0$ there exists $s>0$ such that for $0<r<s$ and $n \geqq N,\left|\int H_{U_{r}}^{(n)}(x, d y) f(y)-H_{D} f(x)\right|<(2\|f\|+1) \varepsilon$.

Proof. Let $\delta>0$ be such that if $\rho(y, z)<\delta$ then $|f(y)-f(z)|<\varepsilon$. From hypothesis 5 ) it is easy to obtain $s>0$ such that

$$
H_{D}(y, E(y, \delta))<\varepsilon
$$

for all $y \in U_{s}$. Now if $0<r<s$ and $n \geqq N$, then since $H_{D} f=H_{D}^{(n)} f$ we have

$$
\begin{aligned}
\left|\int H_{U_{r}}^{(n)}(x, d y) f(y)-H_{D} f(x)\right| & =\left|\int H_{U_{r}}^{(n)}(x, d y) f(y)-\int H_{D}^{(n)}(x, d y) f(y)\right| \\
& =E_{x}^{(n)}\left\{\mid f\left(X\left(T_{U_{r}}\right)\right)-H_{D}^{(n)} f\left(X\left(T_{U_{r}}\right)\right) ; T_{U_{r}}<\infty\right\}
\end{aligned}
$$

by the strong Markov property of $X^{(n)}$. But from (7.2) it is easily seen that the above integrand is less than $\varepsilon+2 \varepsilon\|f\|$.

Proposition 7.4. $H_{D}^{(\infty)}\left(x,{ }^{\circ}\right)=H_{D}(x, \cdot)$ for $x \in K$ and $D \in \mathscr{D}_{\infty}$.

Proof. Let $D$ and $x$ be as above. Let $f \in \mathscr{C}$. Suppose $r>0$ to be such that $P_{x}^{(\infty)}\left[T_{\bar{v}_{r}}<T_{U_{r}}\right]=0$. Let $F_{k}=\left\{y \mid \rho\left(y, \bar{U}_{r}\right) \leqq 1 / k\right\}$. Then $T_{F_{k}} \uparrow T_{\bar{U}_{r}}$ a.s. $P_{x}^{(\infty)}$ as $k \rightarrow \infty$ since $X^{(\infty)}$ is a Hunt process. Let $A=\left\{T_{\bar{U}_{r}}<T_{U_{r}}\right\} \cup\left\{\lim _{k} T_{F_{k}}<T_{\bar{U}_{r}}\right\}$. Then $P_{x}^{(\infty)}(\Lambda)=0$. Define $\phi$ on $\Omega$ by setting $\phi=f\left(X\left(T_{U_{r}}\right)\right)$ if $T_{U_{r}}<\infty$ and $\phi=0$ otherwise. It is easily checked that if $\omega \notin \Lambda$, then $\phi$ is $J_{1}$-continuous at $\omega$. By (7.1) we then have $H_{U_{r}}^{(n)} f(x) \rightarrow H_{U_{r}}^{(\infty)} f(x)$. Now in view of Lemma 7.2 we can choose $r_{m} \downarrow 0$ such that $P_{x}^{(\infty)}\left[T_{\bar{U}_{r_{m}}}<T_{U_{r_{m}}}\right]=0$ for all $m$. Then the above convergence and Lemma 7.3 implies that $H_{U_{r_{m}}}^{(\infty)} f(x) \rightarrow H_{D} f(x)$. Since also $H_{U_{r_{m}}}^{(\infty)} f(x) \rightarrow H_{D} f(x)$ by the quasileft-continuity of $X^{(\infty)}$ and Proposition 7.1, we have $H_{D}^{(\infty)} f(x)=H_{D} f(x)$. The proposition is proved since $f, D$ and $x$ are arbitrary.

Corollary 7.5. $H_{D}^{(\infty)}(x, \cdot)=H_{D}(x, \cdot)$ for $x \in K, D \in \mathscr{D}$.

Proof. Let $D_{n} \in \mathscr{D}_{\infty}$ be such that $D_{n} \downarrow D$. Then for $f \in \mathscr{C}, H_{D_{n}}^{(\infty)} f(x) \rightarrow H_{D}^{(\infty)} f(x)$. On the other hand, from (7.2) we easily obtain $H_{D_{n}} f(x) \rightarrow H_{D} f(x)$. Hence by the previous proposition $H_{D}^{(\infty)} f(x)=H_{D} f(x)$.

Now Theorem 2 is completely proved except for the uniqueness assertion. But this is easy, since if another Hunt process on $K$ has the same hitting distributions on the sets in $\mathscr{D}$ and the same expected lifetime as $X^{(\infty)}$, starting at every $x \in K$, then clearly it has the same resolvent on $\mathscr{C}_{0}$ as $X^{(\infty)}$, and thus by the uniqueness part of the Hille-Yoshida theorem (see the paragraph following Corollary 5.6) it has the same transition operators on $\mathscr{C}_{0}$ as $X^{(\infty)}$ and is therefore equivalent to $X^{(\infty)}$. 


\title{
References
}

1. Blumenthal, R. M., Getoor, R. K.: Markov processes and potential theory. New York and London: Academic Press 1968.

2. - - McKean, H.P., Jr.: Markov processes with identical hitting distributions. Illinois J. Math. 6, 402-420 (1962).

3. Boboc, N., Constantinescu, C., Cornea, A.: Semigroups of transitions on harmonic spaces. Revue Roumaine Math. pur. appl. 12, 763-805 (1967).

4. Bochner, S.: Harmonic analysis and the theory of probability. Berkeley, California: University of California Press (1955).

5. Dawson, D. A.: The construction of a class of diffusions. Illinois J. Math. 8, 657-684 (1964).

6. Hansen, W.: Konstruktion von Halbgruppen und Markoffschen Prozessen. Inventiones math. $\mathbf{3}$, 179-214 (1967).

7. - Charakterisierung von Familien exzessiver Funktionen. Inventiones math. 5, 335-348 (1968).

8. Hunt, G. A.: Markoff processes and potentials, I, II and IV. Illinois J. Math. 1, 44-93, 316-369 (1957); and 2, 151-213 (1958).

9. Knight, F., Orey, S.: Construction of a Markov process from hitting probabilities. J. Math. Mech. 13, 857-873 (1964).

10. Meyer, P.A.: Brelot's axiomatic theory of the Dirichlet problem and Hunt's theory. Ann. Inst. Fourier 13/2, 357-372 (1963).

11. Shih, C.T.: Construction of Markov processes from hitting probabilities, abstract. Notices Amer. math. Soc. 13, 234 (1966).

12. - Markov processes whose hitting distributions are dominated by those of a given process. Trans. Amer. math. Soc. 129, 157-179 (1967).

13. Skorokhod, A.V.: Limit theorems for Markov processes. Theor. Probab. Appl. 3, 202-245 (1958).

14. Stone, $C$.: Weak convergence of stochastic processes defined on semi-infinite time intervals. Proc. Amer. math. Soc. 14, 694-696 (1963).

\author{
Dr. C.T. Shih \\ Dept. of Mathematics \\ University of Michigan \\ Ann Arbor, Michigan 48104 \\ USA
}

(Received February 3,1970) 